\title{
Non-Boltzmann Modeling for Air Shock-Layer Radiation at Lunar-Return Conditions
}

\author{
Christopher O. Johnston ${ }^{*}$ and Brian R. Hollis ${ }^{\dagger}$ \\ NASA Langley Research Center, Hampton, VA, 23681 \\ and \\ Kenneth Sutton \\ National Institute of Aerospace, Hampton, VA, 24060
}

\begin{abstract}
This paper investigates the non-Boltzmann modeling of the radiating atomic and molecular electronic states present in lunar-return shock-layers. The Master Equation is derived for a general atom or molecule while accounting for a variety of excitation and de-excitation mechanisms. A new set of electronic-impact excitation rates is compiled for $\mathrm{N}, \mathrm{O}$, and $\mathrm{N}_{2}{ }^{+}$, which are the main radiating species for most lunar-return shock-layers. Based on these new rates, a novel approach of curve-fitting the non-Boltzmann populations of the radiating atomic and molecular states is developed. This new approach provides a simple and accurate method for calculating the atomic and molecular non-Boltzmann populations while avoiding the matrix inversion procedure required for the detailed solution of the Master Equation. The radiative flux

*Aerospace Engineer, Member AIAA

$\dagger$ Aerospace Engineer, Senior Member AIAA

$¥$ Senior Research Fellow, Associate Fellow AIAA


values predicted by the present detailed non-Boltzmann model and the approximate curve-fitting approach are shown to agree within $5 \%$ for the Fire 1634 s case.

\section{Nomenclature}

$$
\begin{aligned}
& a_{0} \quad=\text { Bohr radius, equal to } 0.529 \times 10^{-8} \mathrm{~cm} \\
& A_{j^{\prime} i^{\prime}} \quad=\text { transition probability for a line with an upper level } j^{\prime} \text { and a lower level } i \text { ' } \\
& c=\text { velocity of light, equal to } 2.997925 \times 10^{10} \mathrm{~cm} / \mathrm{s} \\
& E_{i} \quad=\text { electronic term energy for an atomic level } i\left(\mathrm{~cm}^{-1}\right) \\
& E_{\text {ionize }}=\text { ionization energy of an atomic level } i \\
& E_{l}{ }^{H} \quad=\text { ionization energy of hydrogen, equal to } 109,697 \mathrm{~cm}^{-1} \\
& e \quad=\text { electron charge, equal to } 4.80298 \times 10^{-10} \mathrm{~cm}^{3 / 2} \mathrm{~g}^{1 / 2} / \mathrm{s} \\
& g_{i} \quad=\text { degeneracy for an atomic level } i \\
& h \quad=\text { Planck's constant, equal to } 6.6256 \times 10^{-27} \text { erg-s } \\
& j_{\text {em,line }}=\text { frequency integrated emission coefficient from atomic lines }\left(\mathrm{erg} / \mathrm{cm}^{3} / \mathrm{s} / \mathrm{sr}\right) \\
& k=\text { Boltzmann constant, equal to } 1.38054 \times 10^{-16} \mathrm{erg} / \mathrm{K} \\
& K_{e}(i, j)=\text { electron impact excitation rate from level } i \text { to level } j \text {; the presence of a } d \text { or } c \text { in } \\
& \text { parenthesis refers to a dissociated or ionized level, respectively. } \\
& K_{M}(i, j)=\text { heavy-particle impact excitation rate from level } i \text { to level } j \text {; the presence of a } d \text { or } c \\
& \text { in parenthesis refers to a dissociated or ionized level, respectively. } \\
& m \quad=\text { electron mass, equal to } 9.1091 \times 10^{-28} \mathrm{~g} \text { and the number of electronic states treated for } \\
& \text { a given atom. } \\
& M_{i} \quad=\text { molecular weight of species } i(\mathrm{~kg} / \mathrm{kg}-\mathrm{mol}) \\
& N_{a} \quad=\text { number density of an atom } \\
& N_{i} \quad=\text { number density of an atomic level } i\left(\text { particles } / \mathrm{cm}^{3}\right)
\end{aligned}
$$




$$
\begin{aligned}
& N_{+} \quad=\text { ion number density }\left(\text { particles } / \mathrm{cm}^{3}\right) \\
& N_{e} \quad=\text { electron number density }\left(\text { particles } / \mathrm{cm}^{3}\right) \\
& q_{r}^{-} \quad=\text { wall-directed radiative heat flux, calculated in units of erg } / \mathrm{s} / \mathrm{cm}^{2}, \text { but usually } \\
& \text { presented in units of } \mathrm{W} / \mathrm{cm}^{2}\left(1 \times 10^{-7} \mathrm{~W} / \mathrm{cm}^{2}=1 \mathrm{erg} / \mathrm{s} / \mathrm{cm}^{2}\right) \\
& Q_{x} \quad=\text { partition function of the energy mode } x \text { or an atom } a \text { (nondimensional) } \\
& \bar{r}_{0} \quad=\text { vector defined in Eq. (13) for the Saha decrements in Eq. (12) } \\
& \bar{r}_{00} \quad=\text { vector defined in Eq. (13) for the Saha decrements in Eq. (12) } \\
& \bar{r}_{j} \quad=\text { vector defined in Eq. (13) for the Saha decrements in Eq. (12), } \\
& \text { where } \mathrm{j} \text { represents a level less than or equal to the number of levels that the QSS } \\
& \text { assumption is applied to. } \\
& T_{e} \quad=\text { electronic temperature }(\mathrm{K}) \text {, assumed equal to } T_{v} \text { and } T_{e} \\
& x=\text { number of active electrons for an atomic level } \\
& \Lambda_{j, i} \quad=\text { escape factor for the transition from } i \text { to } j \text { (nondimensional) } \\
& \rho_{i} \quad=\text { refers to the Saha-decrement of level } i \text {, defined by Eq. (8) } \\
& \tau_{\iota} \quad=\text { radiative lifetime for a transition with an upper state } i(\mathrm{~s}) \\
& \text { Subscripts } \\
& e \quad=\quad \text { refers to the electronic energy mode } \\
& \text { EQ = assumes that Boltzmann and Saha equilibrium exists among the electronic levels } \\
& i \quad=\text { refers to the lower electronic state of an atomic transition } \\
& j \quad=\quad \text { refers to the upper electronic state of an atomic transition }
\end{aligned}
$$

\section{Superscripts}

SB = indicates Saha-Boltzmann equilibrium

- $\quad=$ indicates the radiative flux or intensity directed towards the vehicle wall 


\section{Introduction}

In a companion paper $^{1}$ the calculation of the radiative emission and absorption is discussed for the various radiative processes of atoms and molecules. The magnitude of the emission and absorption coefficients from these various radiative processes are proportional to the number density of the emitting or absorbing electronic state. For all of the examples worked by Johnston et al. ${ }^{1}$, it was assumed that these electronic states are populated by a Boltzmann distribution. This assumption is accurate for chemical equilibrium conditions. It has been shown ${ }^{2}$ that there are significant regions of chemical nonequilibrium in the lunar return shock-layers of present interest, which require that the non-Boltzmann electronic state populations be considered. The present paper investigates the calculation of these non-Boltzmann electronic state populations for the significant radiating species present in air shock-layers at lunar return conditions. The species considered in this paper include two atomic species, $\mathrm{N}$ and $\mathrm{O}$, and one molecular species, $\mathrm{N}_{2}{ }^{+}$. Although it was shown by Johnston et al. ${ }^{1}$ that the $\mathrm{NO}$ and $\mathrm{O}_{2}$ molecules contribute to the radiation at lunar-return conditions, it was found that they could be assumed to be populated in a Boltzmann distribution. This assumption is allowable because these molecules do not reach a peak in the nonequilibrium region of the shock layer (unlike the $\mathrm{N}_{2}^{+}$molecule), so their nonequilibrium contribution is small.

A review of past non-Boltzmann modeling for shock-layer radiative heating is discussed in Section II. The definition of the atomic levels for $\mathrm{N}$ and $\mathrm{O}$ used in the present work are discussed in Section III. The atomic and molecular electronic state-populations are modeled in the present work using a collisional-radiative (CR) model, which accounts for the repopulation and depopulation of the atomic and molecular states through collisional and radiative processes. 
These processes are discussed individually in Section IV. In Section V, the general formulation of the CR model's governing equation, called the Master Equation, is derived from the processes discussed in Section IV. A practical method of solving the Master Equation is presented in Section VI. The task of assembling the rates required for the atomic $\mathrm{CR}$ modeling of $\mathrm{N}$ and $\mathrm{O}$ is discussed in Section V. Excitation rates from several sources are compared and a final set of rates is compiled and presented. Based on this detailed rate model and the solution procedure for the Master Equation discussed in Section VI, an "approximate atomic CR" (AACR) model is developed, which allows for the efficient and accurate application of the atomic CR model. The AACR model is presented in Section VIII, and is conveniently represented by curve fits presented in Appendix C of Ref. 18. The non-Boltzmann modeling of the $\mathrm{N}_{2}{ }^{+}$is discussed in Section IX. The influence of various collisional processes on this molecule is studied, and rates from several sources are compared. A recommended set of rates is proposed, and a set of curve fits are constructed for the radiating electronic states of $\mathrm{N}_{2}{ }^{+}$.

\section{Overview of Non-Boltzmann Modeling}

A significant difference between two widely used radiation codes is the treatment of the electronic state population of the atoms and molecules. The NEQAIR ${ }^{3}$ code applies a collisional-radiative (CR), or non-Boltzmann, model for the calculation of the electronic state populations, while the RAD/EQUIL ${ }^{4}$ code assumes a Boltzmann distribution of electronic states. This difference is significant because in regions of chemical and thermodynamic nonequilibrium, the electronic states of atoms and molecules do not follow a Boltzmann distribution. The nonBoltzmann model applied in NEQAIR is based on the approach originated by Bates et al. ${ }^{5}$.

Figure 1 illustrates a typical population distribution of the electronic states for nonequilibrium atomic nitrogen. The populations were calculated using the CR model 
developed in the present work and presented in Section VII. In this figure, $N_{i}$ is the number density of level $i, g_{i}$ is the degeneracy of the level, and $E_{i}$ is the term-energy of the level. It is seen that the CR model predicts much lower number densities for the highly excited states than predicted by the Boltzmann model. This is a characteristic of nonequilibrium conditions during compression, such as those found behind a shock wave in hypersonic flow. The opposite is true for expanding nonequilibrium conditions, such as those found in a favorable pressure-gradient boundary layer with chemically reacting flow. The lower excited state number densities result in decreased radiation, relative to the Boltzmann prediction, from flowfield regions of chemical nonequilibrium.

In the early 1990s, flowfield calculations that included chemical and thermodynamic nonequilibrium became standard. For these flowfields, the RAD/EQUIL code was inadequate because it assumed a Boltzmann distribution of the electronic states, which is incorrect in regions of nonequilibrium. Instead of resorting to the computationally intensive NEQAIR code for these situations, corrective procedures and major revisions to the RAD/EQUIL code were introduced. Gally et al. ${ }^{6,7}$ developed two approximate correction methods, which were implemented in RAD/EQUIL, to account for the non-Boltzmann population of atomic electronic states. The first of these models, referred to as the $1^{\text {st }}$ order local thermodynamic nonequilibrium (LTNE) model, was originally proposed by Carlson ${ }^{8}$. It assumed that the excited atomic states were in equilibrium with the ions and electrons, instead of with the ground electronic state of the atom. The excited state number densities were therefore calculated using the Saha-Boltzmann equation as follows

$$
N_{i}^{S B}=N_{+} N_{e}\left(\frac{h^{2}}{2 \pi m k T_{e}}\right)^{3 / 2} \frac{g_{i} \exp \left[-h c\left(E_{i}-E_{\text {ionize }}\right) / k T_{e}\right]}{2 Q_{+}}
$$

instead of with the regular Boltzmann equation 


$$
N_{i}^{B}=N_{a} \frac{g_{i} \exp \left(-h c E_{i} / k T_{e}\right)}{Q_{a}}
$$

On the other hand, the number densities for the ground states, which include three low-lying states for nitrogen and oxygen, were calculated using Eq. (2). The rationale for such a simple model is apparent from Figure 1, where the results of Eqs. (1) and (2) are compared with the detailed CR model results. It is seen that the three low lying states do follow a Boltzmann distribution closely, and the highly excited states do approach the Saha-Boltzmann population as the ionization limit is approached. The $1^{\text {st }}$ order LTNE method is a very simple method for approximately accounting for the non-Boltzmann population of atomic states. It also shows the connection between chemical nonequilibrium and non-Boltzmann radiation. In regions of chemical equilibrium, Eqs. (1) and (2) are equivalent, by definition, because Eq. (1) is obtained by substituting Eq. (2) into the "law of mass action" equation" for the following reaction

$$
A \leftrightarrow A^{+}+e^{-}
$$

where $A$ represents a neutral atom and $A^{+}$represents its ion. In regions of chemical equilibrium, the flowfield solver will have enforced the law of mass action, which requires for the reaction in Eq. (3) that the neutral species, ion, and electron number densities are related as follows

$$
N_{a}=N_{+} N_{e}\left(\frac{h^{2}}{2 \pi m k T_{e}}\right)^{3 / 2} \frac{Q_{a}}{2 Q_{+}} \exp \left(h c E_{\text {ionize }} / k T_{e}\right)
$$

Substituting this into Eq. (2) makes clear the equality of Eqs. (1) and (2) in regions of chemical equilibrium and explains why, in such a case, all three of the lines in Figure 1 would be the same. A procedure similar to the $1^{\text {st }}$ order LTNE model was also outlined by Geendyke and Hartung ${ }^{10}$, although they applied the method exclusively to a step model representation of the spectrum. The $2^{\text {nd }}$ order LTNE model, also presented by Gally et al. ${ }^{11}$, grouped together the excited states of an atom and treated them as a single species in the flowfield calculation. The populations of the 
various levels of the excited states were then distributed in a Boltzmann distribution, with the number density of the "excited" specie determined from the flowfield calculation. The $2^{\text {nd }}$ order model was shown to result in slightly larger values than the $1^{\text {st }}$ order model, with a difference of about $10 \%$ for the cases considered.

\section{Atomic Level Model}

The emission and absorption of an atomic line is proportional to the number density of the upper and lower electronic state of the defined transition, respectively. If the number density of these states are assumed to follow a Boltzmann distribution, and if the energy and degeneracy of the upper or lower states are defined for each atomic line, then there is no need for a separately defined set of atomic levels. This is true because the Boltzmann relationship and Plank function allow for the emission and absorption of each line to be determined. For the non-Boltzmann case, on the other hand, a separate model is required for calculating the population of the electronic states of the radiating atom. The definition of each atomic state in this calculation must be related to the defined upper and lower state of each atomic line transition. Therefore, in preparation for the discussion of the atomic non-Boltzmann model (in Section VII), this section presents a model for the atomic levels of nitrogen and oxygen suitable for the present hypersonic shock-layer applications.

There are few available guidelines for defining an adequate set of atomic levels for nitrogen and oxygen for the purpose of modeling the radiative heat flux from a chemical nonequilibrium shock-layer. Bourdon and Vervisch ${ }^{12}$ discussed the choice of an energy level model for atomic nitrogen. The focus of their study was the computation of the three-body recombination rate through the use of a CR model. They concluded that the 22 level model presented by Park ${ }^{13}$ and the 14 level model presented by Kunc and Soon ${ }^{14}$ were inadequate and 
proposed instead a 43 level model similar to that used by Park ${ }^{15}$. Bourdon et al. ${ }^{16}$ reached a similar conclusion that 41 levels are required for atomic oxygen. Because the present study is focused on calculating the radiative flux from a shock layer, and not calculating the three-body recombination rate, the conclusion of Bourdon and Vervisch ${ }^{12}$ and Bourdon et al. ${ }^{16}$ that over 40 levels are required for nitrogen and oxygen is not directly applicable. Because no further guidance is available, the current model will be chosen as a compromise between the Bourdon studies and the widely used (for shock layer radiation) Park ${ }^{15}$ model. The new model, which is presented in detail by Johnston ${ }^{18}$, consists of 35 levels for $\mathrm{N}$ and 32 levels for $\mathrm{O}$. The data for these levels were obtained from the NIST database ${ }^{17}$; except for those that group all the levels of a single principal quantum number $(n)$, which were taken from Park's work. For both $\mathrm{N}$ and $\mathrm{O}$, the first 25 levels are ungrouped, while the rest of the levels consist of groups of closely spaced levels. This allows for maximum precision in the CR model calculation for the lower levels, which are the most important for the radiation calculation. It is likely that the total number of levels could be reduced, with a negligible loss in accuracy, by further grouping. Nevertheless, the present groupings will be maintained to provide benchmark results for assessing further simplifications. The relationship between the energy for a grouped level $i$ and a set of ungrouped levels $i$ ' is:

$$
E_{i}=\frac{\sum g_{i^{\prime}} E_{i^{\prime}}}{\sum g_{i^{\prime}}}
$$

and for the degeneracy is

$$
g_{i}=\sum g_{i^{\prime}}
$$


where the summation is over all of the levels $i$ ' in the group $i$. The levels contained in a group are assumed to be populated in a local Boltzmann distribution, which allows the individual level number density $\left(N_{i^{\prime}}\right)$ to be related to the group number density $\left(N_{i}\right)$ as follows

$$
N_{i^{\prime}}=N_{i} \frac{g_{i^{\prime}}}{g_{i}} \exp \left[-\frac{h c}{k T_{e}}\left(E_{i^{\prime}}-E_{i}\right)\right]
$$

This relationship is useful for relating data given in terms of the number density of the individual level (such as atomic line data and electron-impact excitation rates) to the number density of the appropriate grouped level.

\section{Excitation and De-Excitation Mechanisms}

As mentioned previously, the population of the radiating atomic and molecular electronic states required for the calculation of the radiative emission and absorption are calculated by solving the so-called Master Equation. The derivation of this equation requires the specification of the various repopulation and depopulation mechanisms of the atomic or molecular states. The present model will consider electron impact, heavy particle impact, and radiative processes. These processes are defined in Table 1 and discussed in detail by Johnston ${ }^{18}$.

\section{The Master Equation}

The Master Equation is the governing equation for the electronic state populations of atoms and molecules. This differential equation, which must be solved for every state of a radiating atom or molecule, equates the time-rate-of-change of a level's population with all of the populating and depopulating mechanisms listed in Table 1. It is common to express the electronic state populations as a nondimensional population factor $\rho_{i}$, which is defined as

$$
\rho_{i}=\frac{N_{i}}{N_{i}^{S B}}
$$


where $N_{i}$ is the number density of the electronic level $i$ and $N_{i}^{S B}$ is number density of level $i$ predicted by the Saha-Boltzmann equation defined in Eq. (1). This factor is convenient because it forces the values from all levels of a given atom to have similar magnitudes. The Master Equation will be written in terms of this factor. Other non-dimensional quantities that will be useful in the present discussion are defined as follows

$$
\begin{gathered}
\psi_{i}=\frac{N_{i}^{D E}}{N_{i}^{S B}} \\
\chi_{i}=\frac{N_{i}^{S B}}{N_{a}}
\end{gathered}
$$

The Master Equation is derived by equating the time-rate-of-change of level $i$ to the excitation and de-excitation processes presented in Table 1. This results in the following equation:

$$
\begin{aligned}
\frac{\partial N_{i}}{\partial t} & =\left(\frac{\partial N_{i}}{\partial t}\right)_{\text {elec-imp-ex }}+\left(\frac{\partial N_{i}}{\partial t}\right)_{h p-i m p-e x}+\left(\frac{\partial N_{i}}{\partial t}\right)_{\text {elec-imp-ion }} \\
& +\left(\frac{\partial N_{i}}{\partial t}\right)_{\text {elec-imp-dis }}+\left(\frac{\partial N_{i}}{\partial t}\right)_{\text {rad-ex }}+\left(\frac{\partial N_{i}}{\partial t}\right)_{\text {rad-ion }}
\end{aligned}
$$

Eq. (11) represents a set of $m$ differential equations for the $m$ unknown $\rho_{i}$ values. If it is assumed that $\partial N_{i} / \partial t$ is equal to zero, then this set of differential equations reduces to a set of linear algebraic equations for the $\rho_{i}$ values. This is the quasi-steady state (QSS) assumption, which is known to be invalid for the ground state of atoms and molecules ${ }^{19}$. To accommodate this, the Master Equation may be solved using the QSS assumption for all levels except for the first. The population of the first level is then obtained by equating the sum of the level number densities of the species to the total number density of that species, as will be discussed in the next section.

The solution of the Master Equation may be written in matrix form for all of the levels except for the first as follows ${ }^{18}$

$$
\bar{\rho}_{2, m}=\bar{r}_{0}+\bar{r}_{00} \bar{\psi}+\bar{r}_{1} \rho_{1}
$$


where

$$
\begin{aligned}
& \bar{r}_{0}=[M]^{-1} \bar{P} \\
& \bar{r}_{00}=[M]^{-1} \bar{Q} \\
& \bar{r}_{1}=[M]^{-1} \bar{R}_{1}
\end{aligned}
$$

The elements of these vectors and matrices are defined as

$$
\begin{gathered}
M(i, i)=-\left[\sum_{j=1}^{m}\left(K_{e}(i, j)+K_{M}(i, j) \frac{N_{M}}{N_{e}}\right)+K_{e}(i, c)+K_{e}(i, d)+\sum_{j=1}^{i-1} \Lambda_{i, j} \frac{A(i, j)}{N_{e}}\right] \\
M(i, j)=\left(K_{e}(i, j)+K_{M}(i, j) \frac{N_{M}}{N_{e}}+\Lambda_{j, i} A(j, i) \frac{N_{j}^{S B}}{N_{i}^{S B} N_{e}}\right) \quad j>i \\
M(i, j)=\left(K_{e}(i, j)+K_{M}(i, j) \frac{N_{M}}{N_{e}}\right) \quad j<i \\
\bar{P}(i)=-K_{e}(i, c)-\Lambda_{c, i} A(c, i) \frac{N_{+}}{N_{i}^{S B}} \\
\bar{Q}_{j}(i)=-K_{e}(i, d) \\
\bar{R}_{j}(i)=-\left(K_{e}(i, 1)+K_{M}(i, 1) \frac{N_{M}}{N_{e}}\right)
\end{gathered}
$$

With $\bar{r}_{00}=0$, Eq. (12) is equivalent to Eq. (14) presented by Bates et al. ${ }^{5}$. Note that Eqs. (14) to (18) are functions of the following flowfield variables: $N_{X}, N_{Y}, N_{M}, N_{e}, T_{t}$ and $T_{e}$ (the $T_{e}$ dependence is implied through the rate coefficients for the electron-impact processes and $T_{t}$ for the heavy-particle processes). Although $N_{+}, N_{i}^{S B}$, and $N_{i}^{D E}$ appear in these equations, they may be reduced to a $N_{e}, N_{X}, N_{Y}, T_{t}$, and $T_{e}$ dependence using the Saha-Boltzmann equation (Eq. (1)) and detailed balancing ${ }^{13,18}$. For atomic species, it is well known that the heavy-particle impact excitation does not contribute significantly to the excitation and de-excitation processes. Ignoring these terms in Eqs. (14) to (18) for atoms results in these equations being functions of only $N_{e}$ and $T_{e}$ (note that the dissociation processes are not considered for atoms). For molecular species, 
it will be shown that for the lunar-return shock layers of present interest, the heavy particle excitation and electron-impact recombination processes may be ignored as well. This results in Eqs. (14) to (18) also being a function of only $N_{e}$ and $T_{e}$. The significance of these dependencies is that they result in the $\bar{r}_{0}$ and $\bar{r}_{j}$ vectors being functions of only $N_{e}$ and $T_{e}$, which means that these vectors may be conveniently compiled in tables or curve fits in terms of these two variables. Thus, the computationally intensive matrix inversion required to calculate these vectors, shown in Eq. (13), may be avoided. This was recognized by Bates and Kingston ${ }^{20}$, who present tables of $\bar{r}_{0}$ and $\bar{r}_{1}$ for hydrogen plasmas with $0 \leq N_{e} \leq \infty, 250 \leq T_{e} \leq 64000$, and $\Lambda_{j, i}=0$ (optically thick) and 1 (optically thin). The present curve-fits developed for the atoms and molecules of present interest will be discussed in Sections VII and VIII, respectively.

\section{A Simple Method for Calculating $\rho_{1}$}

The previous section presented a method of calculating the $\rho_{i}$ values for energy levels above the first level by making the quasi-steady state approximation $\left(\partial N_{i} / \partial t=0\right)$. Equation (12) shows that these values can be represented as a linear combination of $\rho_{l}$. This section discusses a method of calculating $\rho_{l}$ using the following equation

$$
\sum_{j=1}^{m} N_{j}=N_{a}
$$

which forces the sum of the individual state populations to equal the total number density of the species obtained from the flowfield. This approach, which was introduced by Park ${ }^{21}$, is used in NEQAIR's QSS subroutine ${ }^{3,19,22}$. Rewriting Eq. (19) in terms of $\rho_{i}$ and separating $\rho_{l}$ from the summation results in

$$
\rho_{1} \chi_{1}+\sum_{j=2}^{m} \rho_{j} \chi_{j}=1
$$


Substituting Eq. (12) into this expression and solving for $\rho_{l}$ results in the following

$$
\rho_{1}=\frac{1-\sum_{j=2}^{m}\left[\bar{r}_{0}(j)+\bar{r}_{00}(j) \bar{\psi}(j)\right] \chi_{j}}{\chi_{1}+\sum_{j=2}^{m} \bar{r}_{1}(j) \chi_{j}}
$$

In summary, the solution procedure for this approach involves the following:

1) Obtain the $\bar{r}_{0}, \bar{r}_{00}$, and $\bar{r}_{1}$ vectors either by solving Eq. (13) or from a previously prepared table or curve fit (recall that $\bar{r}_{0}$ and $\bar{r}_{1}$ contain $m$ terms).

2) Calculate $\rho_{l}$ from Eq. (21)

3) Calculate the $\rho_{i}$ values from Eq. (12), which is written for this case as

$$
\rho_{i}=\bar{r}_{0}(i)+\bar{r}_{00}(i) \overline{\psi t}(i)+\bar{r}_{1}(i) \rho_{1}
$$

As mentioned previously, the method outlined here is equivalent to that used by Park in the QSS subroutine of NEQAIR, although in the present case the equations have been formulated in terms of the $\bar{r}_{0}, \bar{r}_{00}$, and $\bar{r}_{1}$ vectors.

\section{Non-Boltzmann Modeling for $\mathbf{N}$ and $\mathbf{O}$}

This section presents rate models, specifically for atomic nitrogen and oxygen, for the significant excitation processes presented in Table 1 and applied in Section V for the Master Equation. The various rates were collected from available experimental and theoretical published sources, compared, and a "best" value chosen. For some processes, no rates were available in the literature, and so approximate formulas were applied. The majority of the effort in constructing this model was obtaining electron-impact excitation rates for transitions between all of the levels of $\mathrm{N}$ and $\mathrm{O}$. This effort will therefore receive the majority of the following discussion regarding the various excitation processes. 


\section{A) Electron-Impact Excitation:}

The process of electron impact excitation was discussed in general in Section IV. The fact that there are electron-impact excitation processes between each atomic level results in a large number of rates being required. For the present study, the 35 levels for $\mathrm{N}$ and 32 levels for $\mathrm{O}$ imply that there are 595 and 496 rates required for each species, respectively. Consequently, collecting this complete set of rates is a difficult and tedious task. The difficulty is compounded by the fact that there are a limited number of detailed calculations and experimental studies for $\mathrm{N}$ and $\mathrm{O}$, which are furthermore limited to a relatively small number of transitions. Thus, extensive use of approximate analytic formulas is required to complete the set of rates for each species. These approximate formulas are discussed in the following paragraph, followed by a discussion of the available detailed rates for both $\mathrm{N}$ and $\mathrm{O}$.

A summary of approximate formulas for the electron-impact cross sections are presented in Table 2. Although these approximate formulas are relatively old, a review of recent astrophysics $^{23,24,24,25,26}$ and plasma physics ${ }^{16,27}$ literature reveals that in the absence of experimental data or detailed calculations, these approximate formulas are the best available alternative. These approximate formulas will be compared with detailed calculations in the following paragraphs. This comparison is aimed at determining the best available rate for each transition, as well as confirming the validity of the approximate formulas for transitions with no detailed rates available. The detailed rate or approximate formula chosen for each transition is presented at the conclusion of this discussion.

The two main sources of detailed quantum mechanical calculations for nitrogen are the recent works by Frost ${ }^{28}$ and Tayal ${ }^{29}$. Frost $^{28}$ presents tabulated values for the transition rates from the lowest three levels $(i=1,2,3)$ to the first 21 levels. These rates were obtained using an R- 
matrix approach and were shown to match available experimental data. Tayal ${ }^{29}$ presents $^{2}$ tabulated values for the transition rates from the lowest three levels $(i=1,2,3)$ to the first 12 levels. An R-matrix approach was also used in this study, although comparisons with experimental results or other calculations were not made. Figure 2 compares the calculations of Frost and Tayal with the approximate formulas summarized in Table 2. Figure 2(a) shows that for the 1-5 forbidden transition, the results of Frost and Tayal differ by an order of magnitude, while the approximate expressions are all similar and fall between Frost's and Tayal's results. Figure 2(b) shows that for transitions between higher levels (3-20), Allen's ${ }^{30}$ approximate formula provides values nearest the values predicted by Frost.

From the above comparison, along with the study of many transitions not discussed above, it was concluded that Van Regmorter's expression is the best available approximate expression for allowed transitions where the upper level of the transition is less than $j=22$. Similarly, Allen's formula was concluded to be the best for forbidden transitions with $j$ less than 22. For transitions with $j$ greater than 22 , Gryzinski's ${ }^{31}$ formula is accurate because of the hydrogenic nature of these upper levels, and was therefore applied. A summary of these chosen rate sources for the final nitrogen model is presented in Table 3. Note that Frost's detailed calculation values were applied where available. These were chosen instead of Tayal's values because Frost considered more transitions, including all of those considered by Tayal.

The main sources of detailed electron-impact excitation rates for atomic oxygen are the studies by Laher and Gilmore ${ }^{32}$, Bhatia and Kastner $^{24}$, and Zatsarinny and Tayal ${ }^{33}$. Laher and Gilmore $^{32}$ obtained rates for transitions from the ground state to the first 19 excited states by reviewing past experimental data and by using a correlation presented by Jackman et al. ${ }^{34}$. Bhatia and Kastner $^{24}$ used a distorted-wave calculation to obtain rates for most of the transitions 
between the lowest ten states. Some of the rates presented by Bhatia and Kastner were taken from Laher and Gilmore, although they were not specified. Zatsarinny and Tayal ${ }^{33}$ calculated values for transitions from the lowest 3 states to the first 21 excited states using an R-matrix approach (with many values missing for transitions from the second and third state). Table 4 lists the sources of the rates chosen for the final oxygen model.

\section{B) Electron-Impact Ionization:}

The process of electron impact ionization is of less importance than electron-impact excitation and bound-bound radiative transitions, and therefore it does not need to be treated as precisely. Also, only one rate is required for each level so that significantly fewer rates are required than the bound-bound processes.

The rate coefficients for electron-impact ionization from excited levels may be calculated accurately with the following formula proposed by Drawin ${ }^{35}$

$$
K(i, c)=1.46 \times 10^{-10} T_{e}^{1 / 2}\left(\frac{E_{1}^{H}}{E_{\text {ionize }}-E_{i}}\right)^{2} \xi y \psi_{1}(y) \quad\left(\mathrm{cm}^{3} \mathrm{~s}^{-1}\right)
$$

where $\xi$ is the number of equivalent electrons ( $\xi=3$ for the ground state of atomic nitrogen and oxygen and $\xi=1$ for all excited states) and $y$ is the reduced energy of the incoming electrons, $y=\left(E_{\text {ionize }}-E_{i}\right) h c / k T_{e}$. The function $\psi_{1}$ is approximated by the following equation

$$
\psi_{1}(y)=\frac{\exp (-y)}{1+y}\left\{\frac{1}{20+y}+\ln \left[1.25\left(1+\frac{1}{y}\right)\right]\right\}
$$

For ionization from the lowest two states of oxygen and nitrogen, the rate coefficients proposed by Kunc and Soon ${ }^{14}$ and Soon and $\mathrm{Kunc}^{36}$ were used.

C) Bound-Bound Radiative Transitions (Atomic Line Transitions): 
The influence of bound-bound radiative transitions on the Master Equation was mentioned in Section IV. This process was also discussed in Section II of Ref. 1, as it related to the spectral modeling of atomic line radiation. The set of atomic lines for nitrogen and oxygen chosen from the NIST database in Ref. 1 are also applied in the present Master Equation model. In general, the transition probabilities are related to the oscillator strengths as follows

$$
A(j, i)=\frac{8 \pi^{2} e^{2}}{m c^{3}} \sum_{i, j^{\prime}} \frac{g_{i^{\prime}}}{g_{j^{\prime}}}\left(w_{C L} c\right)^{2} f_{i^{\prime} j^{\prime}}\left[\frac{g_{j^{\prime}}}{g_{j}} \exp \left(-\frac{h c}{k T_{e}}\left(E_{j^{\prime}}-E_{j}\right)\right)\right] \quad\left(s^{-1}\right)
$$

where the term in brackets accounts for grouped levels, as discussed in Section III. The summation in this equation is over all of the $i$ ' and $j^{\prime}$ individual levels present in the $i$ and $j$ grouped levels. Only optically allowed radiative transitions are considered in the present study. Although Kunc and Soon ${ }^{14}$ and Soon and $\mathrm{Kunc}^{36}$ considered various forbidden transitions, they note that their influence is small, especially the relatively large electron number densities present in the hypersonic shock-layers of interest in this study.

\section{D) Bound-Free Radiative Transitions:}

The bound-free radiative transitions, introduced in Section IV, are of minor importance for most

cases. The approximate expression presented by Drawin ${ }^{35}$, based on the hydrogenic model for the bound-free cross sections, is applied in this study. This is written as follows:

$$
A(c, i)=5.20 \times 10^{-14} Z u_{1}^{3 / 2} G_{i} i^{-3} \bar{K}\left(u_{i}\right) \exp \left(u_{i}\right) \quad\left(\mathrm{cm}^{3} s^{-1}\right)
$$

where the exponential integral is defined as

$$
\bar{K}\left(u_{i}\right)=\int_{t=u_{i}}^{\infty} \frac{\exp (-t)}{t} d t
$$

The detailed bound-free cross-section obtained from the TOPbase could be applied for a more detailed calculation, although this has a negligible influence on the results. 


\section{The Approximate Atomic CR (AACR) Model}

Using the detailed CR model described in the previous section as a baseline, an approximate atomic CR (AACR) model was developed, which is significantly simpler to apply than the detailed CR model and is nearly as accurate at predicting the resulting nonequilibrium radiative emission. The details of this method are presented here and its results compared with the detailed CR model for some relevant shock-layer conditions. Comparisons will also be made to Gally's $1^{\text {st }}$ order LTNE method ${ }^{11}$, which is the approximate method discussed previously in Section II. Recall that the Gally's method assumed that the three lowest levels were in a Boltzmann distribution with the ground state (Eq. (2)) and the upper levels were in a Saha-Boltzmann distribution (Eq. (1)).

The approximate atomic CR (AACR) model developed in this study contains the following three main approximations: 1) the $\bar{r}_{0}$ and $\bar{r}_{1}$ vectors defined in Eq. (13) are modeled as single curve fits over a wide range of $T_{e}$ and $N_{e}$ values, 2) closely spaced atomic states are assumed to be in a Boltzmann distribution with each other, 3) the three lowest atomic states are assumed populated by a Boltzmann distribution. The only approximate aspect of the first of these approximations is that a single curve fit over a range of $T_{e}$ and $N_{e}$ values deviates slightly from the actual curve-fitted data. The dependence of $\bar{r}_{0}$ and $\bar{r}_{1}$ on only $T_{e}$ and $N_{e}$ was pointed out in Section $\mathrm{V}$, and is therefore not an approximation. The second approximation listed above is apparent from Figure 1, which shows groups of closely spaced levels with nearly the same slope (among the levels in the group) as the Boltzmann and Saha-Boltzmann curves. With this assumption, once the population of a single level in each of these groups is known, then the population of the other levels in the group may be calculated from Eq. (7). Table 5 presents the grouping of levels for $\mathrm{N}$ and $\mathrm{O}$, defined in terms of the level model discussed in Section III, that 
were chosen here for the AACR model. The level in each group whose $\bar{r}_{0}$ and $\bar{r}_{1}$ values were curve-fit are also listed in this table. These curve-fits were created from the detailed model discussed the previous section for the following range of conditions: 7,000< $T_{e}<14,000 \mathrm{~K}$ and $1 \times 10^{14}<N_{e}<1 \times 10^{16}$ particles $/ \mathrm{cm}^{3}$. The third assumption that the first three levels are populated by a Boltzmann distribution, which can be shown to be a good approximation for all the conditions of interest in the present study, allows the number densities of the curve-fit level for each group to be written in terms of Eqs. (8) and (22). The procedure for applying the AACR method is as follows:

1) Calculate the $r_{0}$ and $r_{l}$ values for each of the groups listed in Table 5 (except the first group) from the curve fits presented in Appendix C of Ref. 18. These $r_{0}$ and $r_{l}$ values belong specifically to the levels listed in the column "Curve-Fit Level for the Group...".

2) Calculate the number densities for the levels in the first group listed in Table 5 assuming a Boltzmann distribution (Eq. (2)).

3) Calculate the number densities of each curve-fit level using the $r_{0}$ and $r_{l}$ values calculated in Step 1, the ground state number density from Step 2, and Eqs. (8) and (22).

4) Calculate the number densities for the other levels in each group using Eq. (40) and the number densities calculated in Step 3.

To examine the accuracy of the proposed AACR model relative to the detailed atomic CR model and the approximate model proposed by Gally, the radiation for the Fire II 1634 second was calculated using each of these models, along with the radiation properties listed in Ref. 18 (which are slightly different than those in Ref. 1) and an uncoupled LAURA stagnation-line flowfield. The temperature profile for this flowfield is presented in Figure 3. This figure indicates the large region of nonequilibrium present in the shock-layer. The total wall-directed 
radiative flux values resulting from each of the atomic non-Boltzmann models are presented in Figure 4. Also presented is a case where the atoms were assumed to be populated in a Boltzmann distribution. The molecular species are treated in each case using the non-Boltzmann model presented later in this paper. Figure 4 illustrates the significant result that three CR models predict very similar radiative flux values throughout the shock layer, with the AACR and Gally Model within $2 \%$ and $5 \%$ of the detailed CR model, respectively. Although the AACR model produces better agreement with the detailed CR model than the Gally model, it should be noted that the Gally model is significantly simpler conceptually and in application. The importance of applying a non-Boltzmann model, rather than assuming a Boltzmann distribution, is clearly shown in this figure with the nearly $100 \%$ over prediction of the Boltzmann case relative to the various CR model cases. The excessive radiation from the Boltzmann model originates entirely from the region thermochemical nonequilibrium directly behind the shock. The three CR model cases, on the other hand, show that this region contributes only slightly to the radiative flux.

The good agreement between the radiative flux predicted by the two approximate models with that predicted by the detailed CR model was noted in the previous paragraph for the Fire 1634 s case. To investigate this comparison further, two points in the shock layer were studied in detail: a nearly thermochemical equilibrium point at $z=2 \mathrm{~cm}$ and a nonequilibrium point near the shock at $z=4 \mathrm{~cm}$. The temperature and relevant number densities for these points are presented in Table 6.

For the nonequilibrium point at $z=4 \mathrm{~cm}$, Figure 5(a) compares the number densities, divided by the degeneracy, of the excited states predicted by the three models (the lowest three levels are not shown because the result of all three models are indistinguishable and the values are orders of magnitude larger than those shown). The significant under prediction of the excited 
state number densities by the Gally model is apparent in this figure, as well as the excellent agreement of the AACR model with the detailed CR model. The under prediction by Gally's model could have been predicted from Figure 1, which presents the excited state number densities for similar conditions and illustrates the trend that the Saha-Boltzmann values for the excited states, which in Gally's model are assumed equal to the excited state number densities, are lower than the $\mathrm{CR}$ model prediction. Figure 5(b) presents the nitrogen atomic line emission from each level corresponding to the number densities shown in Figure 5(a). The differences in these emission values are consistent with the differences in the number densities of these levels, as they should be since the emission is proportional to the number density of the upper level of the transition. For the detailed CR model case, the total emission from nitrogen lines is 0.065 $\mathrm{W} / \mathrm{cm}^{3}$-sr while the overall emission from all radiative processes is $0.844 \mathrm{~W} / \mathrm{cm}^{3}-\mathrm{sr}$. Thus, the nitrogen lines contribute less than $10 \%$ to the emission (most of the emission at this point is due to molecular band systems), which explains why the radiative flux at $\mathrm{z}=4 \mathrm{~cm}$ in Figure 4 predicted by the Gally model is similar to the other models, even though it was shown in Figure 5(b) that this model significantly under predicted the nitrogen line emission.

The $z=2 \mathrm{~cm}$ point listed in Table 6 is essentially in chemical equilibrium. This means that the Gally model reduces to a Boltzmann distribution (because the Saha-Boltzmann and Boltzmann equations are identical for chemical equilibrium conditions). Figure 5(c) compares the excited states number densities for this case, and it is seen that the population of the lower levels predicted by the Detailed and AACR model are noticeably lower than the Gally prediction. These levels do not reduce to their Boltzmann values because they are strongly radiating, meaning they are rapidly depopulated by spontaneous emission of photons and corresponding transitions to lower levels. The characteristic of being a strongly radiating level is 
a result of being the upper level of atomic line transitions with large transition probabilities, which were defined in Eq. (25). Also, it has been assumed that the gas is optically thin, as it applies to the Master Equation, meaning the escape factors have been set equal to one. This maximizes the influence of radiative transitions on the Master Equation. Figure 5(d) compares the nitrogen atomic line radiation for this case. As expected from the number densities, the Gally model over predicts the emission for the lower states, which are seen to be the most strongly radiating. The total (including all radiating mechanisms of all species) emission from this point for the Detailed CR model case is $2.29 \mathrm{~W} / \mathrm{cm}^{3}$-sr while that from nitrogen line emission is 1.02 $\mathrm{W} / \mathrm{cm}^{3}$-sr. Thus, it is seen that the nitrogen lines contribute a significant fraction of the total emission at this point, unlike for the previous $z=4 \mathrm{~cm}$ point. The Gally model predicts a 1.22 $\mathrm{W} / \mathrm{cm}^{3}$-sr contribution from the nitrogen lines. This larger value explains the larger radiative flux value from the Gally model shown in Figure 4.

From the present discussion regarding the non-Boltzmann modeling of atomic species, it may be concluded that for the shock layer applications of present interest, the AACR model provides a sufficiently accurate non-Boltzmann model that is both computationally efficient and simple to apply. The six grouped levels used by this model for each species (listed in Table 5) essentially means that the 35 and 32 levels used to model $\mathrm{N}$ and $\mathrm{O}$ may be reduced to these six grouped levels.

\section{Non-Boltzmann Modeling for $\mathrm{N}_{2}{ }^{+}$}

For shock layers in air at lunar return conditions and near peak heating (radiative and convective), the $\mathrm{N}_{2}{ }^{+}$and $\mathrm{N}_{2}$ molecules are the main contributors to the nonequilibrium molecular band radiation. The contributions from the NO and $\mathrm{O}_{2}$ bands discussed in Section VI of Ref. 1 are limited to the equilibrium regions of the shock-layer. For lower velocity nonequilibrium 
conditions, such as those studied by Levin et al. ${ }^{37}$, the shock layer temperature is on the order of $6,000 \mathrm{~K}$ and the nonequilibrium contribution from $\mathrm{NO}$ and $\mathrm{O}_{2}$ may be large. For such conditions, the present discussion is not applicable, and instead, the discussions by Levin et al. ${ }^{37}$ and Gorelov et al. ${ }^{38}$ should be consulted. The present section discusses the modeling of only $\mathrm{N}_{2}{ }^{+}$, while a similar discussion is presented by Johnston ${ }^{18}$ for $\mathrm{N}_{2}$.

The rate model for the electronic excitation of $\mathrm{N}_{2}{ }^{+}$, for the lunar return shock layers of interest here, does not require that all of the processes discussed in Section IV be included. Nevertheless, for completeness, all of the processes will be included in the initial model proposed here, except for the electron-impact ionization process, which was shown to be negligible in the preliminary stage of this study (using the rates presented by Teulet ${ }^{39}$ ). Note that the process of electron-impact ionization of molecules is not considered in the kinetic model applied for the flowfield chemistry ${ }^{13}$.

The electronic levels for $\mathrm{N}_{2}{ }^{+}$are shown in Table 7 and the chosen rates for electronimpact excitation, dissociation, heavy-particle excitation, and radiative transitions are shown in Table 8 along with their reference source. The rates are related to the coefficients listed in this table as follows:

$$
K_{r}=a_{r} T_{e}^{b_{r}} \exp \left(-c_{r} / T_{e}\right)
$$

where $K_{r}$ represents $K_{e}(i, j)$ for $r=1$ to $6, K_{e}(i, d)$ for $r=7$ to 10 , and $K_{M}(i, j)$ for $r=11$ and 12 . For many of the rates, the only available values were the theoretical predictions by Teulet et al. ${ }^{39}$. These values were used when no other values were available. Preference was given to other values because the method used by Teulet et al. was more approximate than the experimental and detailed calculation procedures used by other researchers. The most important rate, and fortunately the most frequently studied rate, is that for the electron impact excitation from the 
$\mathrm{N}_{2}{ }^{+}(\mathrm{X})$ state to the $\mathrm{N}_{2}^{+}(\mathrm{B})$ state, which is the second rate listed in Table 8 . This rate is the most significant for determining the population of the $\mathrm{N}_{2}{ }^{+}(\mathrm{B})$ state, which is the upper level of the important $\mathrm{N}_{2}^{+}(1-)$ band system. Figure 6 compares the rates proposed by numerous researchers for this process. The solid lines represent the cases where the actual rates were presented by the researcher, whereas the non-solid lines indicate that the excitation cross-section was presented, which was converted to a rate by numerically integrating the following formula:

$$
K_{e}(i, j)=\frac{8 \pi}{m^{1 / 2}}\left(\frac{1}{2 \pi m k T_{e}}\right)^{3 / 2} \int_{0}^{\infty} \sigma_{i j}(E) \exp \left(-\frac{h c}{k T_{e}} E\right) E d E
$$

This figure indicates the large discrepancy between the various studies, with values ranging many orders of magnitude. Because there is no guidance in choosing the best of these values, the fixed-nuclei result of $\mathrm{Nagy}^{40}$ was chosen because it was the most recent and because it was roughly in the middle of the other predictions. The other sources of rates used for $\mathrm{N}_{2}{ }^{+}$in the present study are Gorelov et al. ${ }^{38}$, Flagan and Appleton ${ }^{41}$, and Chernyi and Losev ${ }^{42}$.

The first topic to examine for the proposed $\mathrm{N}_{2}{ }^{+}$model is the importance of each of the processes listed in Table 8. To aid in this study, it is useful to define the ratio of the inflow and outflow of a level $j$ due to a specific process. For example, the ratio of the outflow from level $j$ due to electron-impact transitions to the total outflow from level $j$ (due to all process) may be written as follows:

$$
\{\text { ratio of } j \text { outflow due to el-imp-trans }\}=\frac{\sum_{j=1}^{m} K_{e}(j, i) N_{j} N_{e}}{\left(\frac{\partial N_{i}}{\partial t}\right)_{\text {outflow }}}
$$

where the total outflow from level $j$ is written as 


$$
\begin{aligned}
\left(\frac{\partial N_{i}}{\partial t}\right)_{\text {outflow }} & =\sum_{j=1}^{m} K_{e}(j, i) N_{j} N_{e}+\sum_{j=1}^{m} K_{M}(j, i) N_{j} N_{M}+K_{e}(c, i) N_{+} N_{e}^{2} \\
& +K_{e}(d, i) N_{X} N_{Y} N_{e}+\sum_{j=i+1}^{m} \Lambda_{j, i} A(j, i) N_{j}+\Lambda_{c, i} A(c, i) N_{+} N
\end{aligned}
$$

The equations for the outflow and inflow from each of the processes discussed in Section IV may be written analogously. We are interested in the emission from the $\mathrm{N}_{2}^{+}(1-)$ band system, which emits from the $\mathrm{N}_{2}^{+}$(B) state, which for the present purposes represents $j$ in Eqs. (30) and (31). Figures 7 and 8 present the inflow and outflow, respectively, for the Fire II 1634 s case using the rates listed in Table 8 . The relative contributors to the inflow, or transition of molecules into the $\mathrm{N}_{2}^{+}$(B) state, are shown in Figure 7 to be dominated by electron-impact transitions from the $\mathrm{N}_{2}{ }^{+}(\mathrm{X})$ state, which is the important process discussed previously ( $r=2$ in Table 8$)$. The other electron-impact transition processes are seen to be much smaller, although not negligible throughout the shock layer. The electron-impact dissociation and heavy-particle impact transitions are seen to only contribute noticeably in narrow regions near the shock and the body. The same trend is apparent in Figure 8, which shows the relative contributors to the outflow, or transition of molecules out of the $\mathrm{N}_{2}^{+}(\mathrm{B})$ state. The influence of radiative transitions is seen to contribute significantly to the outflow. Note that this calculation assumes that the molecular radiation is optically thin, which means that the radiation transitions have their maximum possible influence on the outflow. Figure 9 presents the net inflow, meaning the inflow ratio of Figure 7 minus the outflow ratio of Figure 8, for the present case. Because the quasi-steady state assumption was applied is solving the Master Equation, meaning the right-hand side of Eq. (11) was set to zero, the sum of the various net inflow components equals zero at each point in the shock layer. This figure shows that although the electron-impact inflow and outflow transitions to and from $\mathrm{N}_{2}^{+}(\mathrm{A})$ and $\mathrm{N}_{2}^{+}(\mathrm{C})$ have noticeable components in Figures 7 and 8 , they are of equal magnitude and cancel out to a negligible amount for the net magnitude shown in Figure 9. 
Therefore, they have little influence on the solution of the Master Equation and the resulting state populations.

The conclusion that the electron-impact dissociation and heavy-particle impact transitions do not contribute significantly to the population of the $\mathrm{N}_{2}{ }^{+}(\mathrm{B})$ state is applicable to all of the shock layers examined in this study. If these mechanisms are ignored, then from Eq. (12), the solution of the Master Equation becomes dependent upon only the electron number density and the electronic temperature, as mentioned previously. This dependence on only these two variables allows for a curve-fit to be conveniently constructed for the $\mathrm{N}_{2}{ }^{+}$state populations. These curve-fits were constructed using the present rate model and are presented in Appendix C of Ref. 18 for the four $\mathrm{N}_{2}{ }^{+}$levels listed in Table 7. Unlike the curve-fits for the atomic species, which consisted of fits for $r_{0}$ and $r_{l}$, the number densities of the levels are fit directly for molecules. This is possible for molecules because $r_{0}$ is equal to zero, which as seen from Eqs. (16) and (13) is a result of the electron-impact ionization and bound-free radiation being ignored.

A comparison of the number densities for $\mathrm{N}_{2}{ }^{+}$obtained with the CR model presented here and those obtained with the Boltzmann model are presented in Figure 10 for the Fire II $1634 \mathrm{~s}$ case. The largest deviation from a Boltzmann distribution is seen to be present for the $\mathrm{N}_{2}{ }^{+}(\mathrm{B})$ state. The CR model predicts a much lower number density, which implies that the emission from the $\mathrm{N}_{2}^{+}(1-)$ band is reduced proportionately. Figure 11 illustrated this reduction in emission by comparing the wall directed radiative flux profiles (resulting from all radiation mechanisms) for the Fire II $1634 \mathrm{~s}$ case obtained by assuming a Boltzmann distribution of the $\mathrm{N}_{2}{ }^{+}(\mathrm{B})$ state or by using the present $\mathrm{CR}$ model for this state. This figure also shows the effect of choosing other rate values for the $r=2$ reaction listed in Figure 6. It is seen that the Teulet et al. ${ }^{39}$ value, which was the largest of the rates considered, drives the $\mathrm{N}_{2}{ }^{+}(\mathrm{B})$ state to a Boltzmann distribution, while 
the Gorelov et al. ${ }^{38}$ value, which was the smallest of the rates considered, decreases the flux about $10 \%$ below the present rate value.

\section{Conclusions}

A simplified method of solving the Master Equation for atomic and molecular electronic state populations was presented. A novel approach of curve-fitting the non-Boltzmann population of the radiating atomic and molecular states as a function of the electronic temperature and electron number density was developed. The approximate atomic CR model (AACR) was developed based on this curve-fit treatment. This approach, which reduced the number of atomic levels treated, was shown to provide electronic state number densities within $5 \%$ of the detailed model. A new set of excitation rates was compiled for the significant radiating species in a lunar-return shock-layer, which include $\mathrm{N}, \mathrm{O}$, and $\mathrm{N}_{2}{ }^{+}$. The influence of electron-impact dissociation and heavy-particle impact transitions were found to be negligible for the $\mathrm{N}_{2}{ }^{+}$molecule. The difference between the rate values obtained from various sources was shown, and influence of these values on the resulting radiative flux for the Fire 1634 s case was illustrated.

\section{References}

\footnotetext{
${ }^{1}$ Johnston, C. O., Hollis, B.R., and Sutton, K., "Spectrum Modeling of Air Shock-Layer Radiation at Lunar-Return Conditions," Journal of Spacecraft and Rockets, to be published.

${ }^{2}$ Greendyke, R.B., and Hartung, L.C., "Convective and Radiative Heat Transfer Analysis for the Fire II Forebody," Journal of Spacecraft and Rockets, Vol. 31, pp. 986-992, 1994.

${ }^{3}$ Park, C., “Nonequilibrium Air Radiation (NEQAIR) Program: User's Manual,” NASA TM 86707, 1985.

4 Nicolet, W.E., "Advanced Methods for Calculating Radiation Transport in Ablation-Product Contaminated Boundary Layers,” NASA CR-1656, 1970.

${ }^{5}$ Bates, D.R., Kingston, A.E., and McWhirter, R.W.P., Recombination Between Electrons and Atomic Ions. I. Optically Thin Plasmas," Proceedings of the Royal Society, Series A, Vol. 267, pp. 297-312, 1962.

${ }^{6}$ Gally, T. A., and Carlson, L. A., "Nonequilibrium Chemical and Radiation Coupling, Part II: Results for AOTV Flowfields," Journal of Thermophyics and Heat Transfer, Vol. 6, pp. 392-399, July-Sept 1992
} 
${ }^{7}$ Gally, T.A., Carlson, L.A., and Green, D., "Flowfield Coupled Excitation and Radiation Model for Nonequilibrium Reacting Flows," Journal of Thermophysics and Heat Transfer, Vol. 7, pp. 285-293, 1993.

8 Carlson, L.A., "Approximations for Hypervelocity Nonequilibrium Radiating, Reacting, and Conducting Stagnation Regions," Journal of Thermophysics and Heat Transfer, Vol. 3, pp. 380-388, 1989.

9 Anderson, J.D., Hypersonic and High Temperature Gas Dynamics, American Institute of Aeronautics and Astronautics, Reston, Virginia, pp. 446-448, 2000.

${ }^{10}$ Greendyke, R.B., and Hartung, L.C., "Approximate Method for the Calculation of Nonequilibrium Radiative Heat Transfer," Journal of Spacecraft and Rockets, Vol. 28, pp. 165-171, 1991.

${ }^{11}$ Gally, T.A., Development of Engineering Methods for Nonequilibrium Radiative Phenomena about Aeroassisted Entry Vehicles, Ph.D. Dissertation, Texas A\&M University, 1992.

${ }^{12}$ Bourdon, A., and Vervisch, P., "Three-Body Recombination Rate of Atomic Nitrogen in Low-Pressure Plasma Flows," Physical Review E, Vol. 54, pp. 1888-1898, 1996.

${ }^{13}$ Park, C., Nonequilibrium Hypersonic Aerothermodynamics, Wiley, New York, pp. 126-129, 1990.

${ }^{14}$ Kunc, J.A., and Soon, W.H., "Collisional-Radiative Nonequilibrium in Partially Ionized Atomic Nitrogen," Physical Review A, Vol. 40, pp. 5822-5843, 1989.

${ }^{15}$ Park, C., "Spectral Line Intensities in a Nonequilibrium Nitrogen Plasma," Journal of Quantitative Spectroscopy and Radiative Transfer, Vol. 8, pp. 1633-1653, 1968.

${ }^{16}$ Bourdon, A., Teresiak, Y., and Vervisch, P., "Ionization and Recombination Rates of Atomic Oxygen in HighTemperature Air Plasma Flows,” Physical Review E, Vol. 57, pp. 4684-4692, 1998.

${ }^{17}$ Ralchenko, Yu, et al., "NIST Atomic Spectra Database, Version 3.1.0," National Institute of Standards and Technology (NIST) Physics Lab, http://physics.nist.gov/PhysRefData/ASD/index. html, July 2006 [cited 3 Sept. 2006].

${ }^{18}$ Johnston, C. O., Nonequilibrium Shock-Layer Radiative Heating for Earth and Titan Entry, Ph.D. Dissertation, Virginia Tech, 2006.

${ }^{19}$ Park, C., "Calculation of Nonequilibrium Radiation in AOTV Flight Regimes," AIAA Paper 84-0306, 1984.

${ }^{20}$ Bates, D.R., and Kingston, A.E., "Properties of a Decaying Plasma," Planetary and Space Science, Vol. 11, pp. 122, 1963.

${ }^{21}$ Park, C., "Calculation of Radiative Properties of Nonequilibrium Hydrogen Plasma," J. Quant. Spectrosc. Radiat. Transfer, Vol. 22, pp. 101-112, 1979.

${ }^{22}$ Whiting, E. E., et al., "NEQAIR96, Nonequilibrium and Equilibrium Radiative Transport and Spectra Program: User's Manual," NASA RP-1389, 1996.

${ }^{23}$ Przybilla, N., et al., "Non-LTE Line Formation for Neutral Oxygen: Model Atom and First Results on A-Type Stars," Astronomy and Astrophysics, Vol. 359, pp. 1085-1106, 2000.

${ }^{24}$ Bhatia, A.K., and Kastner, S.O., "The Neutral Oxygen Spectrum. I. Collisionally Excited Level Populations and Line Intensities under Optically Thin Conditions," The Astrophysical Journal Supplement Series, Vol. 96, pp. 325$341,1995$. 
${ }^{25}$ Fisher, V., Bernshtam, V., Golten, H., Maron, Y., "Electron-Impact Excitation Cross-Sections for Allowed Transitions in Atoms," Physical Review A, Vol. 53, pp. 2425-2431, 1996.

${ }^{26}$ Lemke, M., and Venn, K.A., "The Interesting Problem of the NLTE Nitrogen Abundance in Vega," Astronomy and Astrophysics, Vol. 309, pp. 558-569, 1996.

${ }^{27}$ Bourdon, A., and Vervisch, P., "Three-Body Recombination Rate of Atomic Nitrogen in Low-Pressure Plasma Flows," Physical Review E, Vol. 54, pp. 1888-1898, 1996.

${ }^{28}$ Frost, R.M., et al., "Calculated Cross Sections and Measured Rate Coefficients for Electron-Impact Excitation of Neutral and Singly Ionized Nitrogen,” Journal of Applied Physics, Vol. 84, pp. 2989-3003, 1998.

${ }^{29}$ Tayal, S.S., "Effective Collision Strengths for Electron Impact Excitation of N I," Atomic Data and Nuclear Data Tables, Vol. 76, pp. 191-212, 2000.

${ }^{30}$ Allen, C.W., Astrophysical Quantities, London, 1962.

${ }^{31}$ Gryzinski, M., "Classical Theory of Electronic and Ionic Inelastic Collisions” Physical Review, Vol. 115, pp. 374$383,1959$.

${ }^{32}$ Laher R.R., and Gilmore, F.R., "Updated Excitation and Ionization Cross Sections for Electron Impact of Atomic Oxygen," Journal of Physical and Chemical Reference Data, Vol. 19, pp. 277-305, 1990.

33 Zatsarinny, O., and Tayal, S.S., "Electron Collisional Excitation Rates for O I using the B-Spline R-Matrix Approach,” The Astrophysical Journal Supplement Series, Vol. 148, 2003, pp. 575-582.

${ }^{34}$ Jackman, C.H., Garvey, R.H., and Green, A.E.S., "Electron Impact on Atmospheric Gases I. Updated Cross Sections," Journal of Geophysical Research, Vol. 82, pp. 5081-5090, 1977.

${ }^{35}$ Drawin, H.W., "Collision and Transport Cross Sections," in Plasma Diagnostics (ed. Lochte-Holtgreven), pp. 842-876, 1968.

${ }^{36}$ Soon, W.H., and Kunc, J.A., “Thermal Nonequilibrium in Partially Ionized Atomic Oxygen," Physical Review A, Vol. 41, pp. 825-843, 1990.

${ }^{37}$ Levin, D.A., Candler, G.V., Erdman, P.W., Zipf, E.C., Epsy, P., Howlett, C., "Flight Measurements of LowVelocity Bow Shock Ultraviolet Radiation," Journal of Thermophysics and Heat Transfer, Vol. 7, pp. 30-36, 1993.

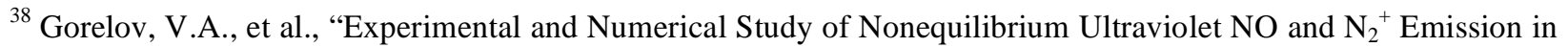
a Shock-Layer," Journal of Thermophysics and Heat Transfer, Vol. 12, pp. 172-179, 1998.

${ }^{39}$ Teulet, P., Sarrette, J.P., and Gomes, A.M., "Calculation of Electron-Impact Inelastic Cross-Sections and Rate Coefficients for Diatomic Molecules. Application to Air Molecules," Journal of Quantitative Spectroscopy and Radiative Transfer, Vol. 62, pp. 549-569, 1999.

${ }^{40}$ Nagy, O., "Excitation Cross-Sections of $\mathrm{N}_{2}{ }^{+}$Molecular Ion by Electron Impact and the Vibrational Energy Levels of the Three Target States, Chemical Physics, Vol. 286, pp. 109-114, 2003.

41 Flagan, R.C., and Appleton, J.P., "Excitation Mechanisms of the Nitrogen First-Positive and First-Negative Radiation at High-Temperature," Journal of Chemical Physics, Vol. 56, pp. 1163-1173, 1972.

${ }^{42}$ Chernyi, G.G., and Losev, S.A., Analysis of Russian Experimental Investigations for Validation of Kinetic Models for Hypersonic Flows in Thermochemical Nonequilibrium, Moscow State University - Institute of Mechanics, pp. 233, 1993. 
${ }^{43}$ Van Regemorter, H., "Rate of Collisional Excitation in Stellar Atmospheres," Astrophysical Journal, Vol. 136, pp. 906-915, 1962.

${ }^{44}$ Park, C., "Comparison of Electron and Electronic Temperatures in a Recombining Nozzle Flow of Ionized Nitrogen - Hydrogen Mixture. Part 1. Theory," Journal of Plasma Physics, Vol. 9, pp. 187-215, 1973. 


\section{Tables}

Table 1. Excitation processes accounted for in the present treatment of the Master Equation

\begin{tabular}{|c|c|c|}
\hline Process & Schematic & Rate of Production (particles- $\mathrm{cm}^{-3}-\mathrm{s}^{-1}$ ) \\
\hline $\begin{array}{l}\text { Electron-Impact } \\
\text { Excitation }\end{array}$ & $X_{i}+e^{-} \Leftrightarrow X_{j}+e^{-}$ & $\left(\frac{\partial N_{i}}{\partial t}\right)_{\text {elec-imp-ex }}=\sum_{j=1}^{m} K_{e}(j, i) N_{j} N_{e}-\sum_{j=1}^{m} K_{e}(i, j) N_{i} N_{e}$ \\
\hline $\begin{array}{l}\text { Heavy Particle } \\
\text { Impact Excitation }\end{array}$ & $X_{i}+M \Leftrightarrow X_{j}+M$ & $\left(\frac{\partial N_{i}}{\partial t}\right)_{h p-i m p-e x}=\sum_{j=1}^{m} K_{M}(j, i) N_{j} N_{M}-\sum_{j=1}^{m} K_{M}(i, j) N_{i} N_{M}$ \\
\hline $\begin{array}{l}\text { Electron-Impact } \\
\text { Ionization }\end{array}$ & $X_{i}+e^{-} \Leftrightarrow X^{+}+e^{-}+e^{-}$ & $\left(\frac{\partial N_{i}}{\partial t}\right)_{\text {elec-imp-ion }}=K_{e}(c, i) N_{+} N_{e}^{2}-K_{e}(i, c) N_{i} N_{e}$ \\
\hline $\begin{array}{l}\text { Electron-Impact } \\
\text { Dissociation }\end{array}$ & $X Y_{i}+e^{-} \Leftrightarrow X+Y+e^{-}$ & $\left(\frac{\partial N_{i}}{\partial t}\right)_{\text {elec-imp-dis }}=K_{e}(d, i) N_{X} N_{Y} N_{e}-K_{e}(i, d) N_{i} N_{e}$ \\
\hline $\begin{array}{l}\text { Bound-Bound } \\
\text { Radiative }\end{array}$ & $X_{j} \Leftrightarrow X_{i}+h v$ & $\left(\frac{\partial N_{i}}{\partial t}\right)_{r a d-e x}=\sum_{j=i+1}^{m} \Lambda_{j, i} A(j, i) N_{j}-\sum_{j=1}^{i-1} \Lambda_{i, j} A(i, j) N_{i}$ \\
\hline $\begin{array}{l}\text { Bound-Free } \\
\text { Radiative }\end{array}$ & $X_{+}+e^{-} \Leftrightarrow X_{i}+h v$ & $\left(\frac{\partial N_{i}}{\partial t}\right)_{\text {rad-ion }}=\Lambda_{c, i} A(c, i) N_{+} N_{e}$ \\
\hline
\end{tabular}

Table 2. Summary of Approximate Equations for Electron-Impact Excitation Rates

\begin{tabular}{ccccc}
\hline & Name & Transition Type & Eq. in Ref. 18 & Ref. \\
\hline 1 & Van Regmorter & allowed & $(4.30)$ & 43 \\
2 & Park approx. & forbidden & $(4.30)$ times 0.4 & 44 \\
3 & Gryzinski & any & $(4.32)-(4.34)$ & 31 \\
4 & Allen & forbidden & $(4.31)$ & 30 \\
\hline
\end{tabular}

Table 3. Selection of Electron-Impact Excitation Rates for $\mathrm{N}$

1) Frost's rates are used for $i=1,2,3$ and $j=2$ through 21

2) Remaining allowed transitions with $j<22$ use Van Regmorter's formula

3) Remaining forbidden transitions with $j<22$ use Allen's formula

4) All remaining transitions use Gryzinski's formula

Table 4. Selection of Electron-Impact Excitation Rates for O

1) Zatsarinny and Tayal rates are used for $i=1,2,3$ and $j=2$ through 21 where available

2) Bhatia and Kastner for remaining rates with $j<10$ where available

3) Gordillo and Kunc for remaining rates with $j<7$

4) Remaining allowed transitions with $j<22$ use Van Regmorter's formula where available

5) Remaining forbidden transitions with $j<22$ use Allen's formula

6) All remaining transitions use Gryzinski's formula 
Table 5. Level Groupings for the AACR Model

\begin{tabular}{ccccc}
\hline Group \# & $\begin{array}{c}\text { Range of Levels for } \\
\text { N from Table 3.1 of } \\
\text { Ref. 18 }\end{array}$ & $\begin{array}{c}\text { Curve-Fit } \\
\text { Level for the } \\
\text { Group for } \boldsymbol{N}\end{array}$ & $\begin{array}{c}\text { Range of Levels for } \\
\text { O from Table 3.2 } \\
\text { of Ref. 18 }\end{array}$ & $\begin{array}{c}\text { Curve-Fit } \\
\text { Level for the } \\
\text { Group for } \boldsymbol{O}\end{array}$ \\
\hline 1 & $1-3$ & - & $1-3$ & - \\
2 & $4-6$ & 5 & $4-7$ & 5 \\
3 & $7-13$ & 10 & $8-13$ & 10 \\
4 & $14-21$ & 17 & $14-21$ & 17 \\
5 & $22-27$ & 24 & $22-27$ & 24 \\
6 & $28-35$ & 28 & $28-35$ & 28 \\
\hline
\end{tabular}

Table 6. Conditions for two points in the Fire $1634 \mathrm{~s}$ shock-layer

\begin{tabular}{ccc}
\hline & $z=2 \mathrm{~cm}$ & $z=4 \mathrm{~cm}$ \\
\hline$T_{e}(\mathrm{~K})$ & 10,467 & 12,670 \\
$T_{t}(\mathrm{~K})$ & 10,459 & 19,186 \\
number densities $\left(\# / \mathrm{cm}^{3}\right)$ & \\
$\mathrm{N}$ & $1.74 \times 10^{16}$ & $1.2 \times 10^{16}$ \\
$\mathrm{~N}^{+}$ & $3.90 \times 10^{15}$ & $3.5 \times 10^{14}$ \\
$\mathrm{e}^{-}$ & $4.55 \times 10^{15}$ & $4.7 \times 10^{14}$ \\
\hline
\end{tabular}

Table 7. $\mathrm{N}_{2}{ }^{+}$electronic levels treated in the present model ${ }^{39}$

\begin{tabular}{ccc}
\hline Level & $E_{e}\left(\mathrm{~cm}^{-1}\right)$ & $g_{e}$ \\
\hline $\mathrm{X}^{2} \Sigma_{\mathrm{g}}^{+}$ & 0.0 & 2 \\
$\mathrm{~A}^{2} \Pi_{\mathrm{u}}$ & $9,016.4$ & 4 \\
$\mathrm{~B}^{2} \Sigma_{\mathrm{u}}^{+}$ & $25,461.5$ & 2 \\
$\mathrm{C}^{2} \Sigma_{\mathrm{u}}^{+}$ & $64,542.0$ & 2 \\
\hline
\end{tabular}


Table 8. Non-Boltzmann rate model for $\mathrm{N}_{2}{ }^{+}$

\begin{tabular}{cccccc}
\hline$r$ & Process & $a_{r}$ & $b_{r}$ & $c_{r}$ & Ref. \\
\hline 1 & $N_{2}^{+}(X)+e^{-} \Leftrightarrow N_{2}^{+}(A)+e^{-}$ & $7.1 \times 10^{-11}$ & 0.0 & 13,300 & 38 \\
2 & $N_{2}^{+}(X)+e^{-} \Leftrightarrow N_{2}^{+}(B)+e^{-}$ & $2.0 \times 10^{-11}$ & 0.73 & 36,649 & 40 \\
3 & $N_{2}^{+}(X)+e^{-} \Leftrightarrow N_{2}^{+}(C)+e^{-}$ & $6.6 \times 10^{-9}$ & 0.41 & 85,038 & 39 \\
4 & $N_{2}^{+}(A)+e^{-} \Leftrightarrow N_{2}^{+}(B)+e^{-}$ & $1.0 \times 10^{-9}$ & 0.0 & 23,500 & 38 \\
5 & $N_{2}^{+}(A)+e^{-} \Leftrightarrow N_{2}^{+}(C)+e^{-}$ & $1.3 \times 10^{-7}$ & 0.11 & 78,403 & 39 \\
6 & $N_{2}^{+}(B)+e^{-} \Leftrightarrow N_{2}^{+}(C)+e^{-}$ & $3.9 \times 10^{-9}$ & 0.34 & 49,622 & 39 \\
\hline 7 & $N_{2}^{+}(X)+e^{-} \Leftrightarrow N+N^{+}+e^{-}$ & $8.02 \times 10^{-31}$ & 5.54 & 101,117 & 39 \\
8 & $N_{2}^{+}(A)+e^{-} \Leftrightarrow N+N^{+}+e^{-}$ & $8.27 \times 10^{-26}$ & 4.38 & 88,142 & 39 \\
9 & $N_{2}^{+}(B)+e^{-} \Leftrightarrow N+N^{+}+e^{-}$ & $2.58 \times 10^{-32}$ & 5.81 & 64,328 & 39 \\
10 & $N_{2}^{+}(C)+e^{-} \Leftrightarrow N+N^{+}+e^{-}$ & $1.31 \times 10^{-28}$ & 4.93 & 35,906 & 39 \\
\hline \multicolumn{7}{c}{} & & & 12,978 & 40 \\
\hline 11 & $N_{2}^{+}(X)+N_{2} \Leftrightarrow N_{2}^{+}(A)+N_{2}$ & $3.8 \times 10^{-2}$ & -2.33 & 41 \\
12 & $N_{2}^{+}(X)+N_{2} \Leftrightarrow N_{2}^{+}(B)+N_{2}$ & $1.9 \times 10^{-2}$ & -2.33 & 36,600 & \\
\hline \multicolumn{7}{c}{$A_{r}\left(\mathrm{~s}^{-1}\right)$} & & & 42 \\
\hline 14 & $N_{2}^{+}(A) \Rightarrow N_{2}^{+}(X)+h v$ & $6.7 \times 10^{4}$ & & & \\
15 & $N_{2}^{+}(B) \Rightarrow N_{2}^{+}(X)+h v$ & $1.5 \times 10^{7}$ & & & 42 \\
\hline
\end{tabular}

\section{Figures}

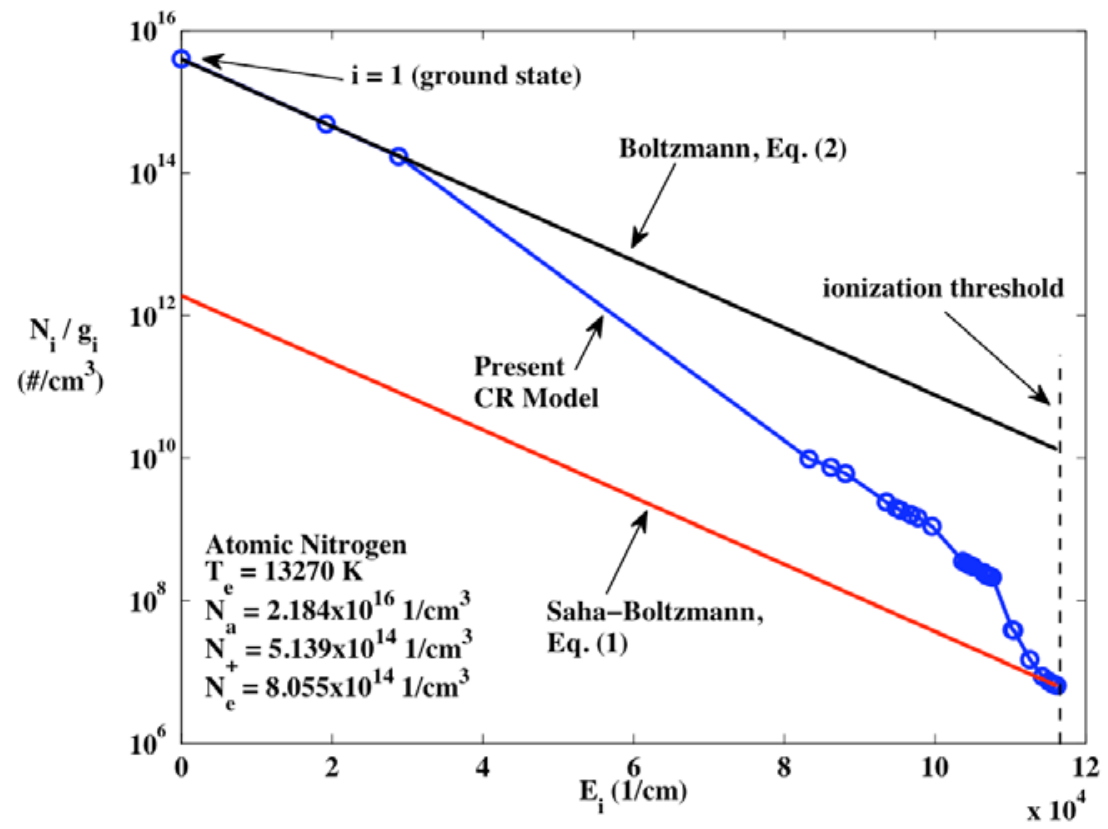

Figure 1. Population of the electronic states of atomic nitrogen in nonequilibrium 


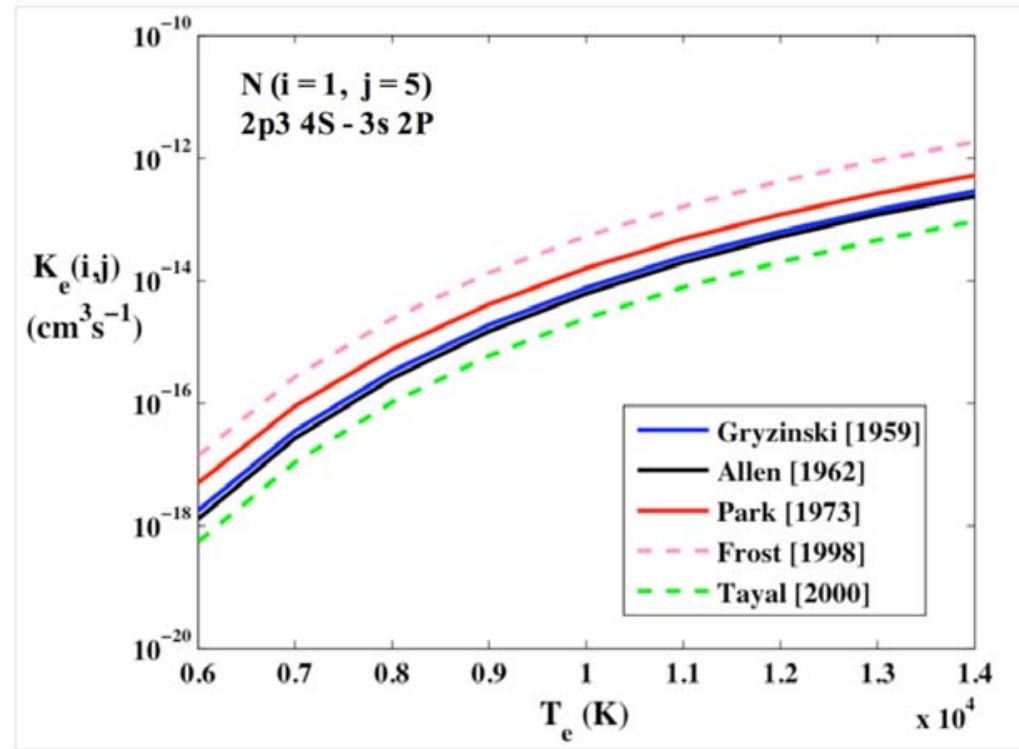

a) Forbidden transition between low $\mathrm{N}$ levels

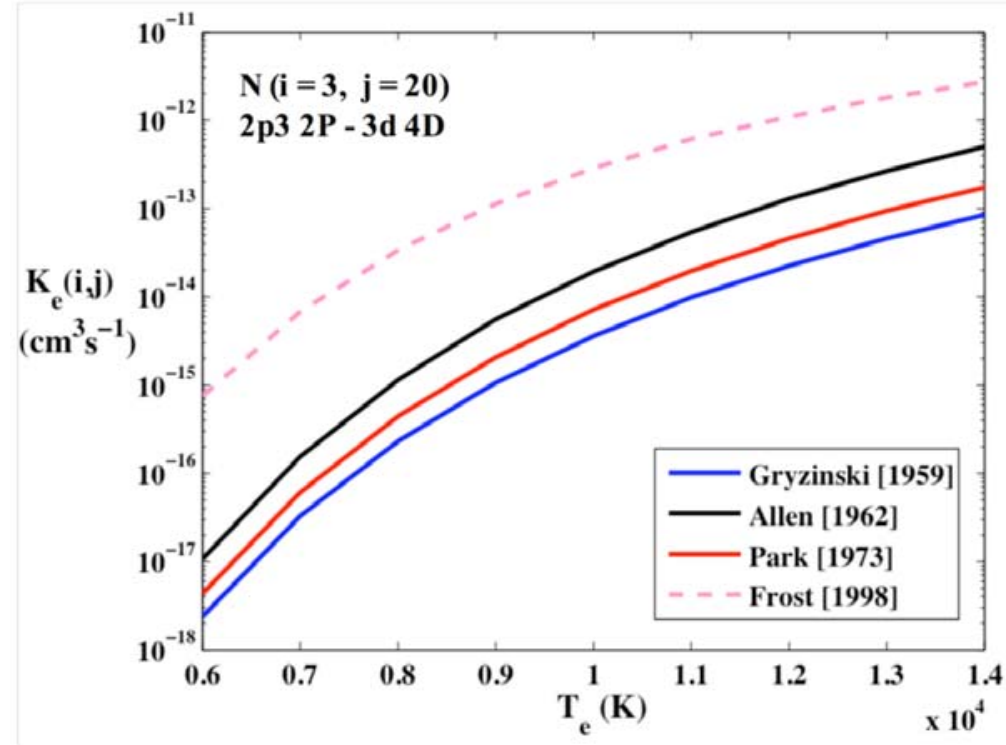

b) Forbidden transition between a high and low $\mathrm{N}$ level

Figure 2. Comparison of the various approximate electron-impact excitation cross-sections with detailed calculations. 


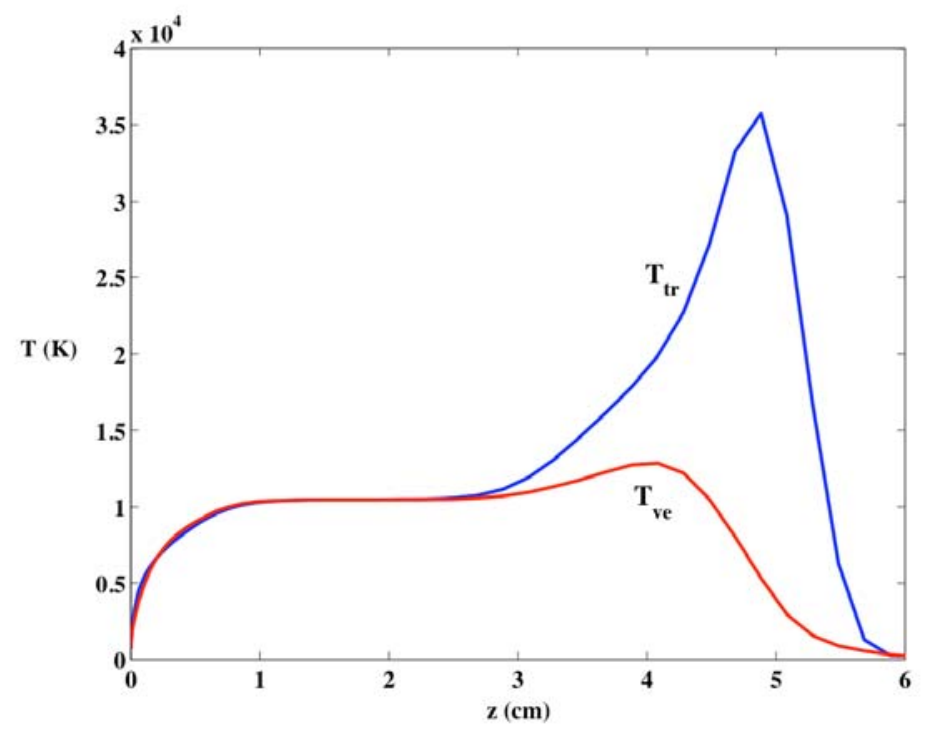

Figure 3. Temperature profile along the stagnation-line predicted by the LAURA code for the Fire 1634 s case.

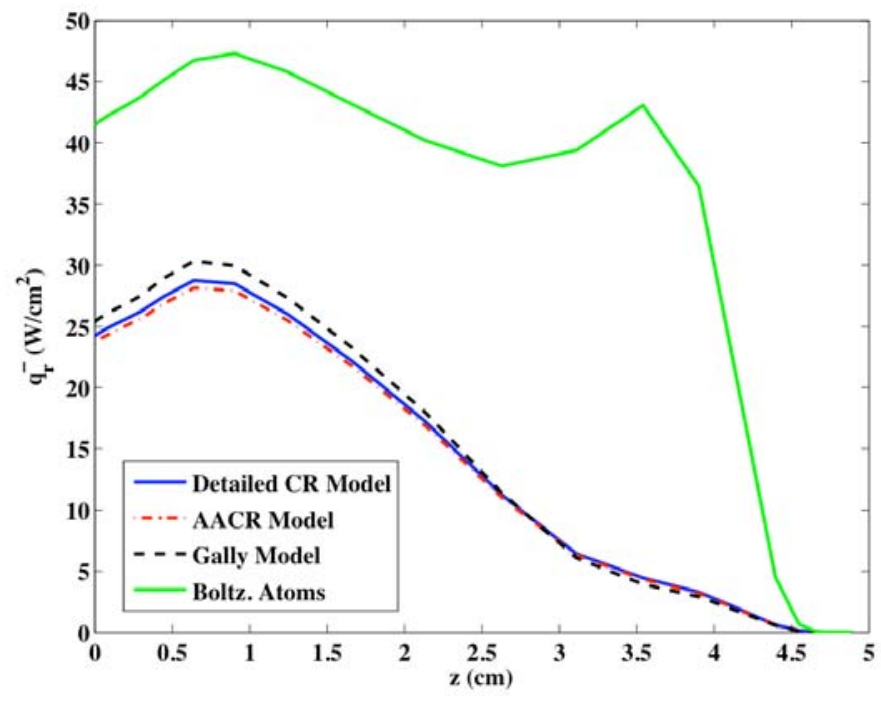

Figure 4. Influence of the various atomic CR models on the total wall-directed radiative flux for the Fire $1634 \mathrm{~s}$ case, the molecules are treated in all cases with the non-Boltzmann model presented later in this paper. 


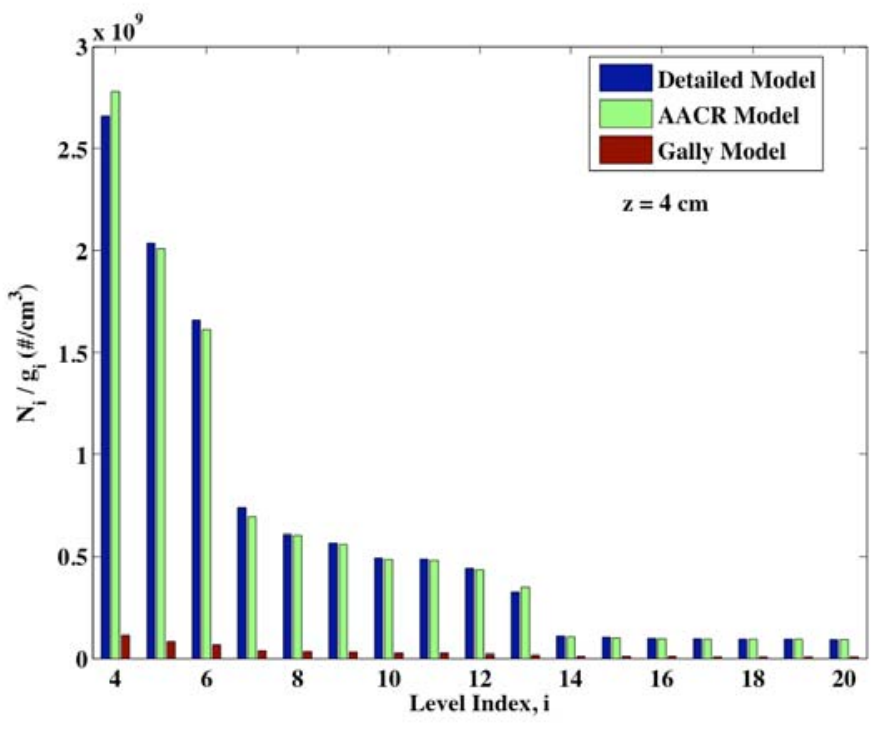

a) Number densities at $\mathrm{z}=4 \mathrm{~cm}$

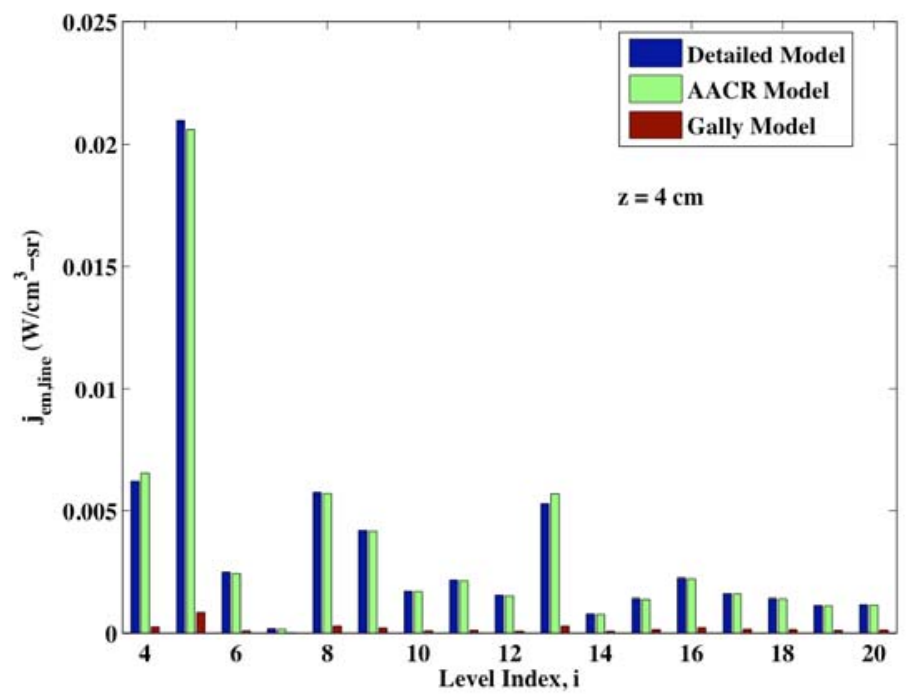

b) Line emission at $\mathrm{z}=4 \mathrm{~cm}$ 


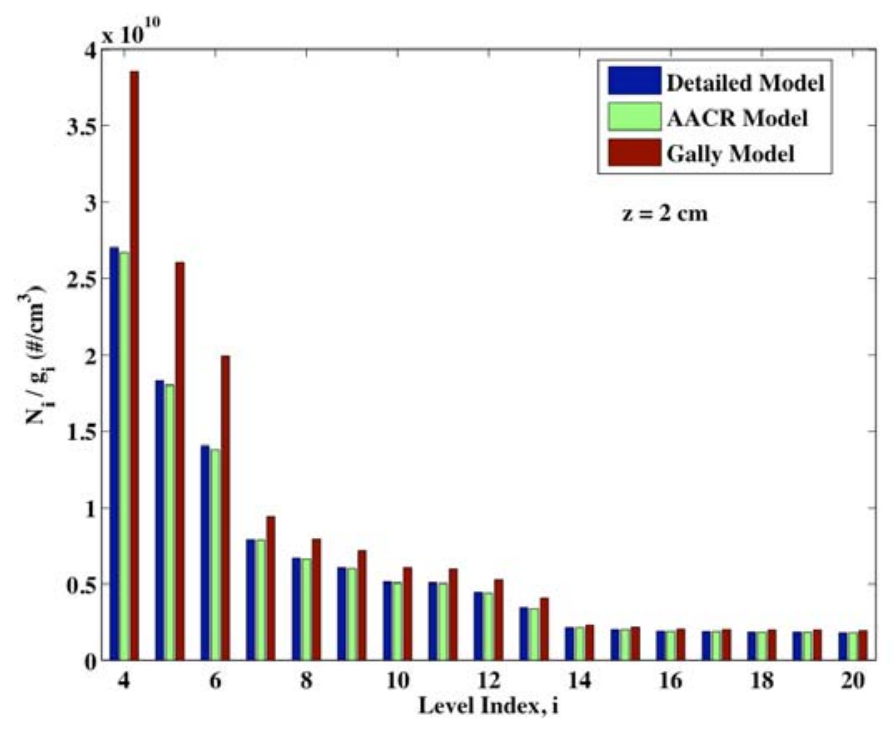

c) Number densities at $\mathrm{z}=2 \mathrm{~cm}$

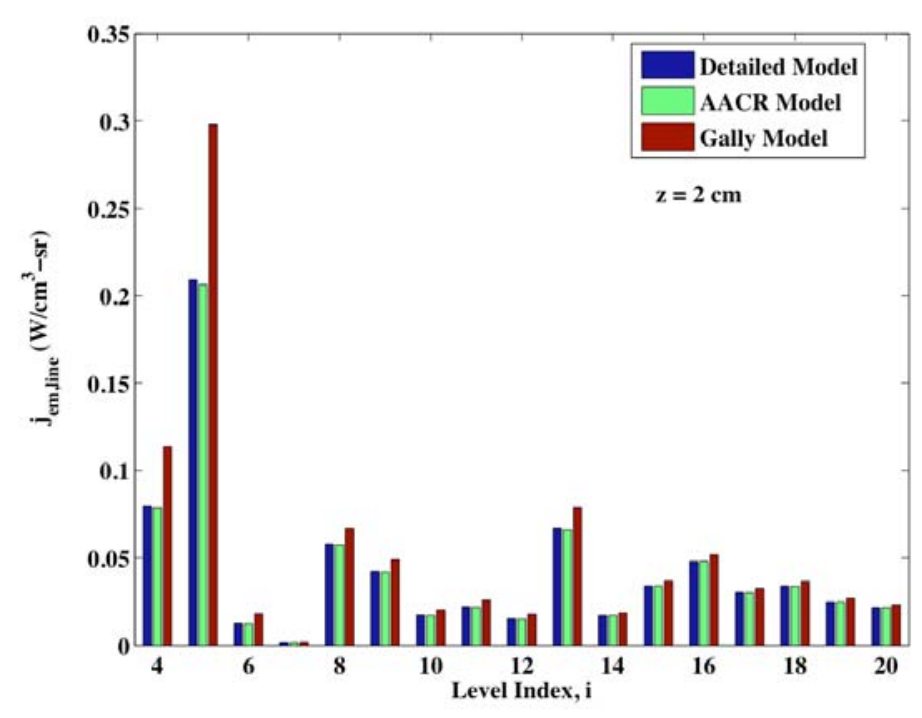

d) Line emission at $\mathrm{z}=2 \mathrm{~cm}$

Figure 5. Number densities and line emission from atomic nitrogen levels predicted by the various models. 


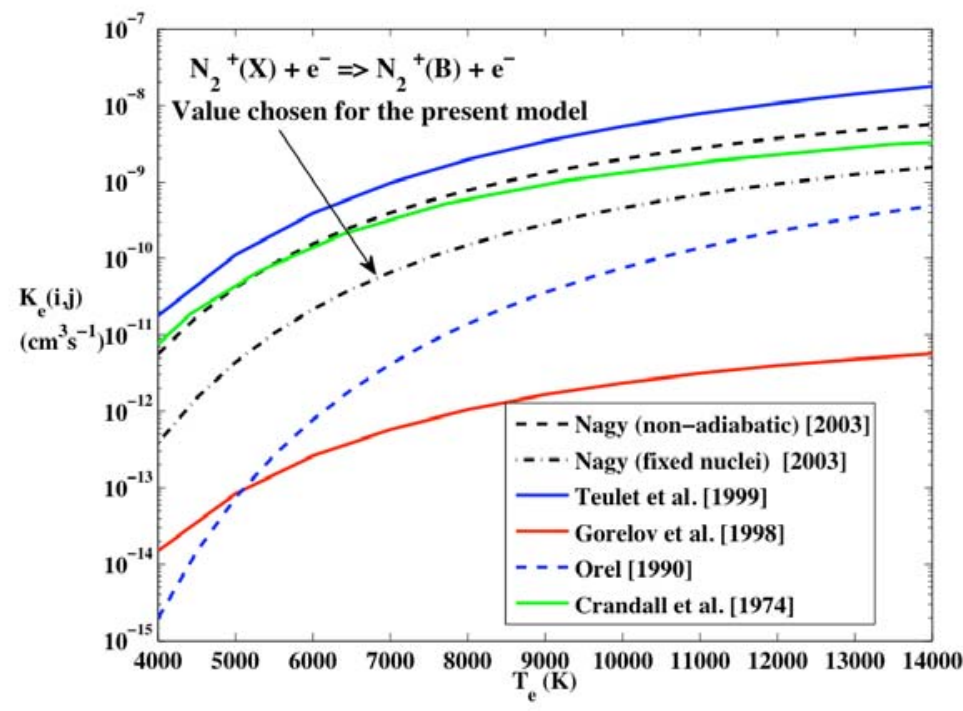

Figure 6. Comparison of electron-impact excitation rates for populating the $\mathrm{N}_{2}{ }^{+}(\mathrm{B})$ state.

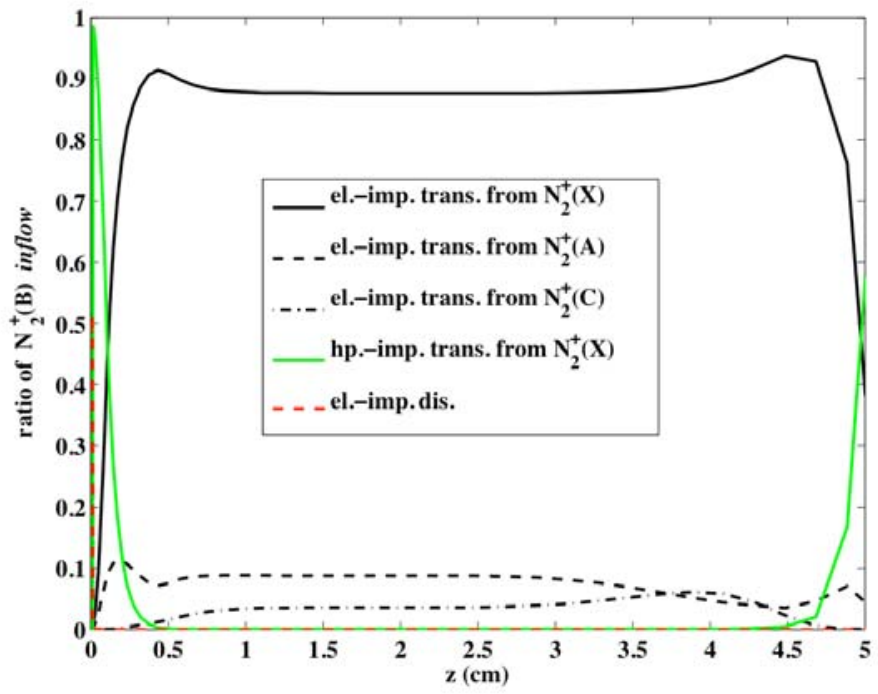

Figure 7. The ratio of $\mathrm{N}_{2}^{+}(\mathrm{B})$ inflow for various process for the Fire II $1634 \mathrm{~s}$ case, using the Table 8 rates. 


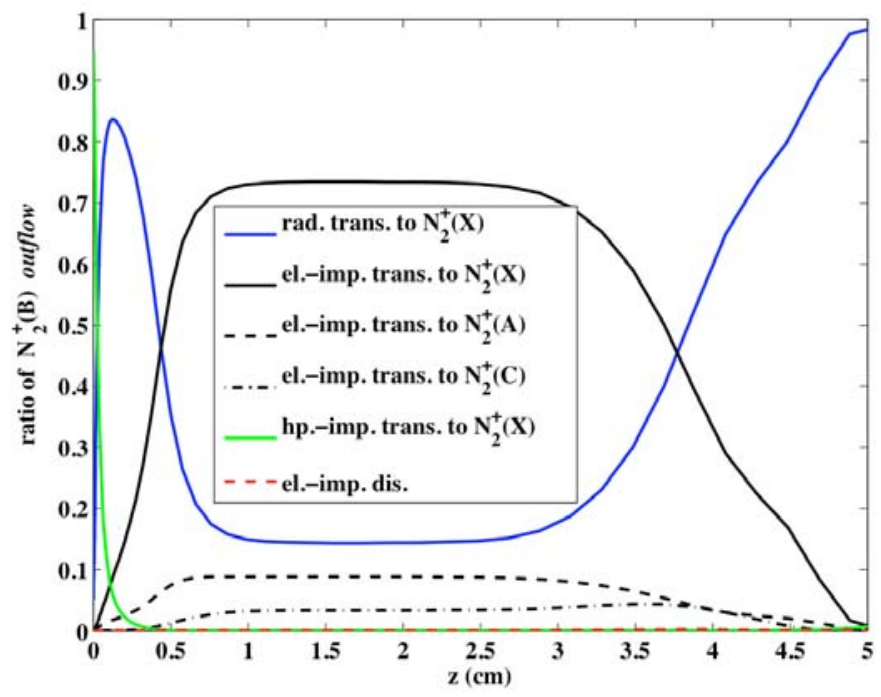

Figure 8. The ratio of $\mathrm{N}_{2}^{+}$(B) outflow for various process for the Fire II $1634 \mathrm{~s}$ case, using the Table 8 rates.

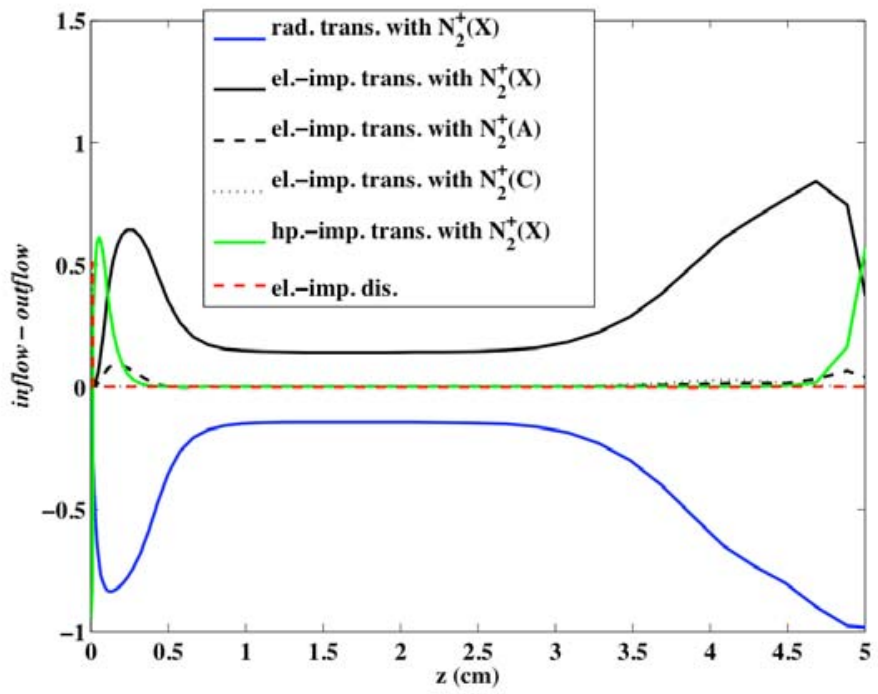

Figure 9. The ratio of $\mathrm{N}_{2}^{+}(\mathrm{B})$ inflow ratio minus the outflow ratio for each process corresponding to Figures 7 and 8. 


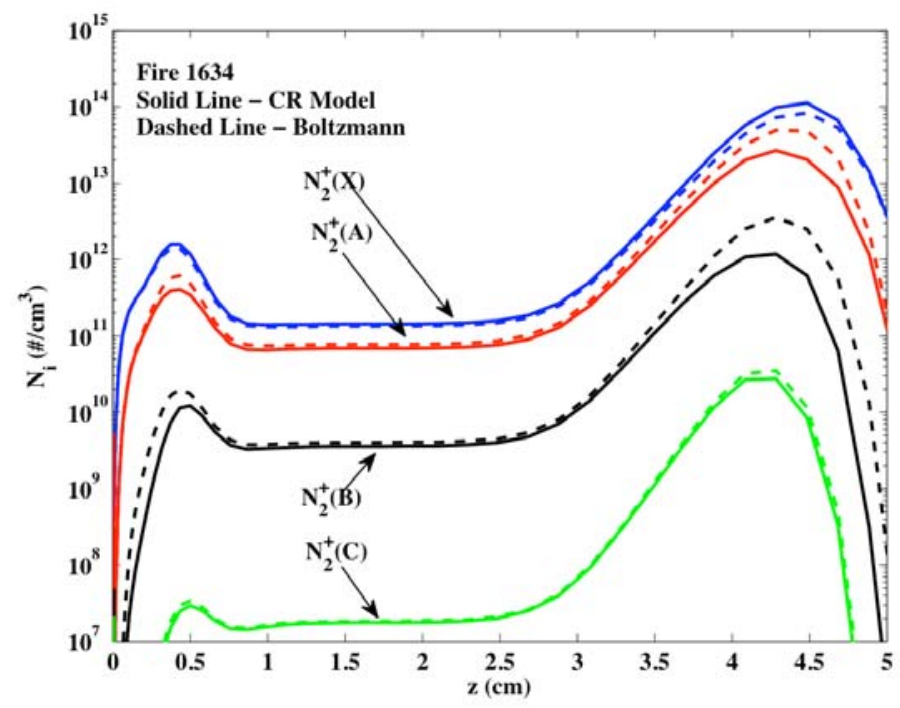

Figure 10. Comparison of the electronic state number densities predicted by the Boltzmann and CR Model along the stagnation line for the Fire II 1634 s case

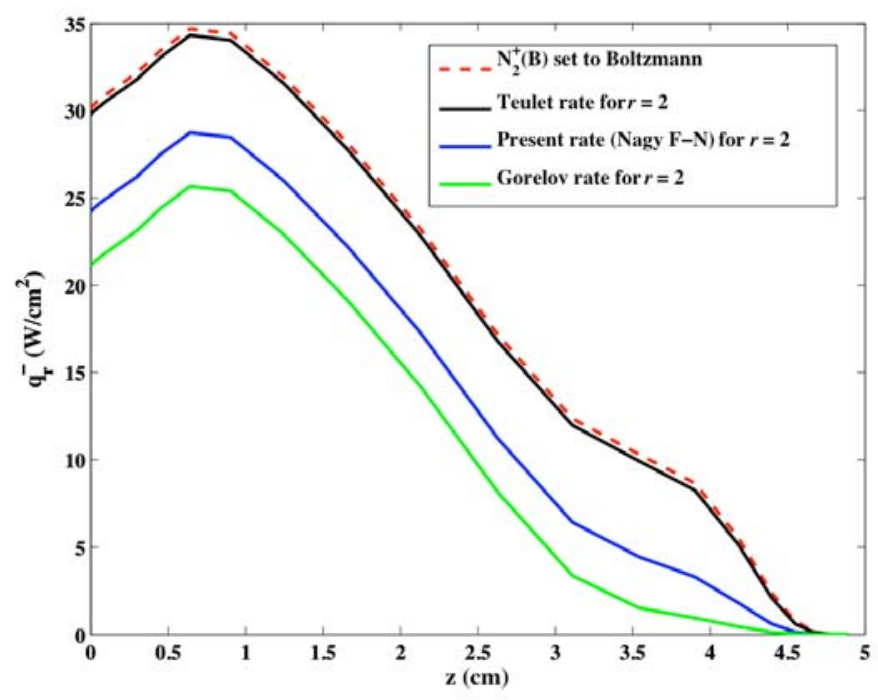

Figure 11. Wall directed radiative flux profiles for the Fire II 1634 case for different treatments of the $\mathrm{N}_{2}{ }^{+}(\mathrm{B})$ state population 